\title{
Ubiquitylation of p62/sequestosome1 activates its autophagy receptor function and controls selective autophagy upon ubiquitin stress
}

Hong Peng ${ }^{1,2,3, *}$, Jiao Yang ${ }^{1,2,3, *}$, Guangyi $\mathrm{Li}^{1,2}$, Qing You ${ }^{1,2}$, Wen Han ${ }^{1}$, Tianrang $\mathrm{Li}^{1}$, Daming Gao , Xiaoduo Xie ${ }^{1}$, Byung-Hoon Lee ${ }^{4}$, Juan $\mathrm{Du}^{5}$, Jian Hou ${ }^{5}$, Tao Zhang ${ }^{6}$, Hai Rao ${ }^{7}$, Ying Huang ${ }^{3}$, Qinrun $\mathrm{Li}^{1}$, Rong Zeng ${ }^{1}$, Lijian $\mathrm{Hui}^{3}$, Hongyan Wang ${ }^{1}$, Qin Xia ${ }^{8}$, Xuemin Zhang ${ }^{8}$, Yongning $\mathrm{He}^{3}$, Masaaki Komatsu', Ivan Dikic ${ }^{10}$, Daniel Finley ${ }^{4}$, Ronggui $\mathrm{Hu}^{1}$

${ }^{I}$ Key Laboratory of Systems Biology, CAS Center for Excellence in Molecular Cell Science, Innovation Center for Cell Signaling Network, ${ }^{2}$ Graduate School, University of Chinese Academy of Sciences; ${ }^{3}$ Institute of Biochemistry and Cell Biology, Chinese Academy of Sciences, 320 Yueyang Road, Shanghai 200031, China; ${ }^{4}$ Department of Cell Biology, Harvard Medical School, 240 Longwood Ave, Boston, MA 02115, USA; ${ }^{5}$ Department of Hematology, Changzheng Hospital, The Second Military Medical University, 415 Fengyang Road, Shanghai 200003, China; ${ }^{6}$ Department of Laboratory Medicine, Huashan Hospital, Fudan University, 12 Central Urumqi Road, Shanghai 200040, China; ${ }^{7}$ Department of Molecular Medicine, University of Texas Health Science Center at San Antonio, San Antonio, Texas 78229, USA; ${ }^{8}$ State Key Laboratory of Proteomics, National Center of Biomedical Analysis, Institute of Basic Medical Sciences, Beijing 100850, China; ${ }^{9}$ Department of Biochemistry, School of Medicine Niigata University, 757, Ichibancho, Asahimachidori, Chuo-ku, Niigata 951-8510, Japan; ${ }^{10}$ Molecular Signaling, Institute of Biochemistry II, Goethe University School of Medicine, 60590 Frankfurt am Main, Germany

Alterations in cellular ubiquitin (Ub) homeostasis, known as Ub stress, feature and affect cellular responses in multiple conditions, yet the underlying mechanisms are incompletely understood. Here we report that autophagy receptor p62/sequestosome-1 interacts with E2 Ub conjugating enzymes, UBE2D2 and UBE2D3. Endogenous p62 undergoes E2-dependent ubiquitylation during upregulation of $\mathrm{Ub}$ homeostasis, a condition termed as $\mathrm{Ub}^{+}$stress, $^{-}$ that is intrinsic to Ub overexpression, heat shock or prolonged proteasomal inhibition by bortezomib, a chemotherapeutic drug. Ubiquitylation of $\mathbf{p 6 2}$ disrupts dimerization of the UBA domain of p62, liberating its ability to recognize polyubiquitylated cargoes for selective autophagy. We further demonstrate that this mechanism might be critical for autophagy activation upon $\mathrm{Ub}^{+}$stress conditions. Delineation of the mechanism and regulatory roles of p62 in sensing Ub stress and controlling selective autophagy could help to understand and modulate cellular responses to a variety of endogenous and environmental challenges, potentially opening a new avenue for the development of therapeutic strategies against autophagy-related maladies.

Keywords: autophagy receptor; ubiquitylation; heat shock; dynamic light scattering; ubiquitin; selective autophagy Cell Research (2017) 27:657-674. doi:10.1038/cr.2017.40; published online 21 March 2017

\section{Introduction}

Ubiquitin (Ub) is a 76-amino-acid polypeptide ubiquitously distributed in all tissues of eukaryotic organisms

\footnotetext{
*These two authors contributed equally to this work. Correspondence: Ronggui Hu

E-mail: coryhu@sibcb.ac.cn

Received 21 July 2016; revised 6 December 2016; accepted 22 January 2017; published online 21 March 2017
}

[1]. Ubiquitylation, the reaction of attaching Ub to a substrate protein or to Ub itself, regulates the stability, function, localization and protein-protein interactions of the substrate [2]. Intracellular Ub (both free and in conjugates) is estimated to be at a level of $\sim 500 \mathrm{pmol} / \mathrm{mg}$ cell lysate [3], making Ub one of the most abundant proteins in a typical cell. Maintaining the cellular pools of $\mathrm{Ub}$ conjugates and free $\mathrm{Ub}$ constitutes an essential part of cellular Ub homeostasis, which is subjected to highly dynamic but strict regulation [4-6]. 
Alteration in Ub homeostasis, termed as Ub stress, is critically implicated in many important biological, pathological or therapeutic conditions such as heat shock, aging, microbial infection, neurodegenerative diseases or chemotherapy [7-9]. Yet another example is that, upon mitochondrial depolarization, an interplay between Pink 1 and Parkin leads to change in the landscape of ubiquitylome surrounding mitochondria and beyond [1012]. Herein, we further specify $\mathrm{Ub}^{+}$stress as a condition of repletion or excess of free $\mathrm{Ub}, \mathrm{Ub}$ conjugates or both. Reciprocally, $\mathrm{Ub}^{-}$stress defines those conditions where the amount of $\mathrm{Ub}$ is insufficient.

$\mathrm{Ub}^{+}$stress is an intrinsic event in cellular responses to variety of pathophysiological conditions, including heat shock that has been shown to upregulate the expression of $\mathrm{Ub}, \mathrm{E} 1 \mathrm{Ub}$-activating enzyme (UBA1) and E2 Ub-conjugating enzymes (such as UBE2D2 or UBE2D3), resulting in an increased level of Ub conjugates $[7,9,13]$. Elevated levels of Ub conjugates are associated with aging [14] and multiple types of neurological diseases [8]. Another typical condition for $\mathrm{Ub}^{+}$stress is prolonged proteasomal inhibition by proteasomal inhibitors, e.g., bortezomib (BTZ), in which the levels of both poly-Ub conjugates and free $\mathrm{Ub}$ accumulate. Given the fact that BTZ is also a chemotherapeutic drug used for treating multiple myeloma [15], it would be of critical importance to understand the molecular mechanisms of cellular responses to BTZ as this might prove to be clinically beneficial.

Meanwhile, manipulation of the cellular level of Ub, although practiced worldwide on daily basis and assumed to marginally impact the cell functions, has also been shown to cause significant changes in many aspects of cellular activities. Overexpression of $\mathrm{Ub}$ in yeast led to elevated tolerance of osmostress, ethanol and canavanine, but sensitized the cells to arsenate and paromomycin [16]. Furthermore, overexpression of Ub caused defects in tissue development in flies [17], and conferred hyper-proliferation and some cancer stem cell-like properties upon mammalian cells $[18,19]$. In contrast, Ub depletion sensitized cells to puromycin-induced proteotoxic stress [20,21]. A decrease in the pool of free Ub through ablation of the $U b b[22,23], U C H-L 1$ [24] or USP 14 [25, $26]$ genes was shown to cause neuronal degenerative disorder-like phenotypes in mice, whereas depletion of $U b c$ led to defective development of the mouse fetal liver [27]. Although Ub stress has significant impact on how cells respond to diverse stimuli, its underlying mechanisms remain poorly understood.

Macroautophagy (hereafter referred to as autophagy) is an evolutionarily conserved process that cells employ to target cytosolic proteins for lysosomal degradation [28,
29]. Cytoplasmic proteins were found to be selectively taken up into nascent autophagic vesicles through a family of autophagy receptors, which commonly bear a functionally conserved LC3-interacting region (LIR) domain [30]. The LIR domain anchors the autophagy receptor to ATG8 family proteins on the surface of the nascent autophagic vesicles [31]. Currently known autophagy receptors include p62/sequestosome-1 (p62/SQSTM1, hereafter referred to as p62), NBR1, NDP52, Nix, Cbl, Stbd1, OPTN and Tollip [30, 32]. Among them, p62 and NBR1 can either work independently or as hetero-oligomers that form via their N-terminal PB1 (Phox and Be$\mathrm{m} 1 \mathrm{p})$ domains and cooperate to recruit target cargoes to the autophagosome [33] to regulate cell signaling, death and metabolism [34]. However, the UBA domain of unmodified p62 tends to form an intermolecular dimer that spatially occludes Ub binding $[35,36]$. It is intriguing to ask what mechanism might activate the unmodified p62 from this closed and auto-inhibited conformation in which binding to the ubiquitylated cargoes is prevented.

\section{Results}

\section{Ubiquitylated p62/SQSTM1 accumulates upon prolonged proteasomal inhibition}

As demonstrated before [37], proteasomal inhibition by prolonged treatment with BTZ $(1 \mu \mathrm{M})$ led to significant accumulation of Ub conjugates (Figure 1A). An upregulation in the level of p62 protein was also observed, in spite of the supposedly faster turnover of p62 upon autophagy activation. This might be primarily attributed to the augmented transcription of the $p 62$ gene mediated by BTZ-stabilized Nrf2 [37, 38]. Upon BTZ treatment, lipidation of LC3 was also increased (Figure 1A). Using Lysotracker Red as a marker [39], the puncta formed by GFP-LC3 were shown to predominantly co-localize with lysosomes in HeLa cells overexpressing $\mathrm{Ub}$ (Supplementary information, Figure S1A), indicating that the LC3-positive dots were indeed associated with lysosomes, rather than protein aggregates formed upon $\mathrm{Ub}^{+}$stress. Furthermore, in $p 62^{-1-}$ mouse embryonic fibroblast (MEF) cells, the level of lipidated LC3 seemed to be unaffected by BTZ treatment but significantly elevated ( $\sim 50 \%$ increase) upon treatment with bafilomycin (BAF, $200 \mathrm{nM}$ ), an inhibitor of vacuolar-type $\mathrm{H}^{+}$-ATPase that prevents the maturation of autophagic vacuoles. This response seemed not to be changed when cells were treated with both BTZ and BAF (Figure 1B). These data suggested that lipidated LC3 is a substrate for autophagy rather than proteasome-mediated degradation. However, when human p62 was re-introduced into these cells, BAF treatment alone increased the lipidated LC3 to $\sim 80 \%$, 
A

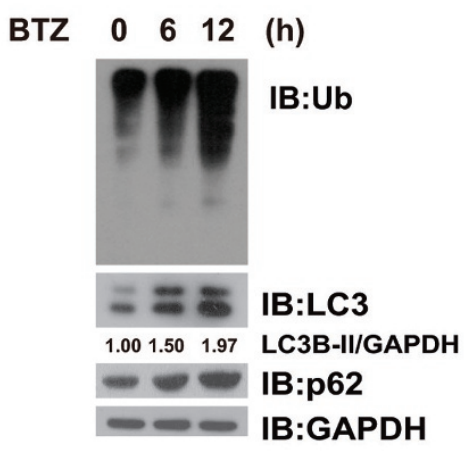

B

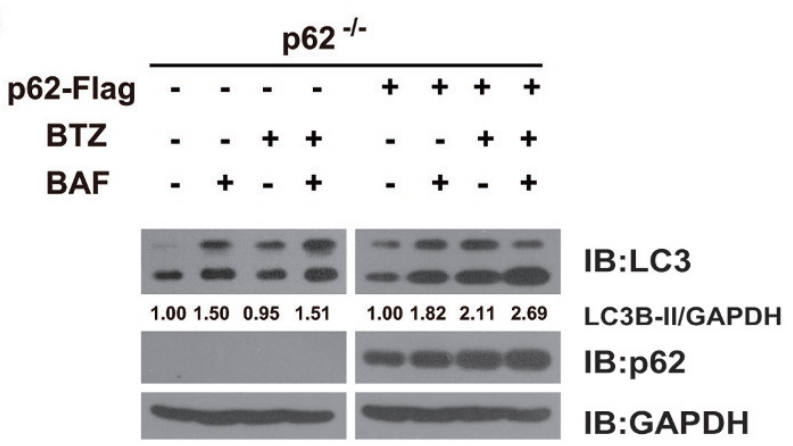

C GFP-LC3 DAPI Merge
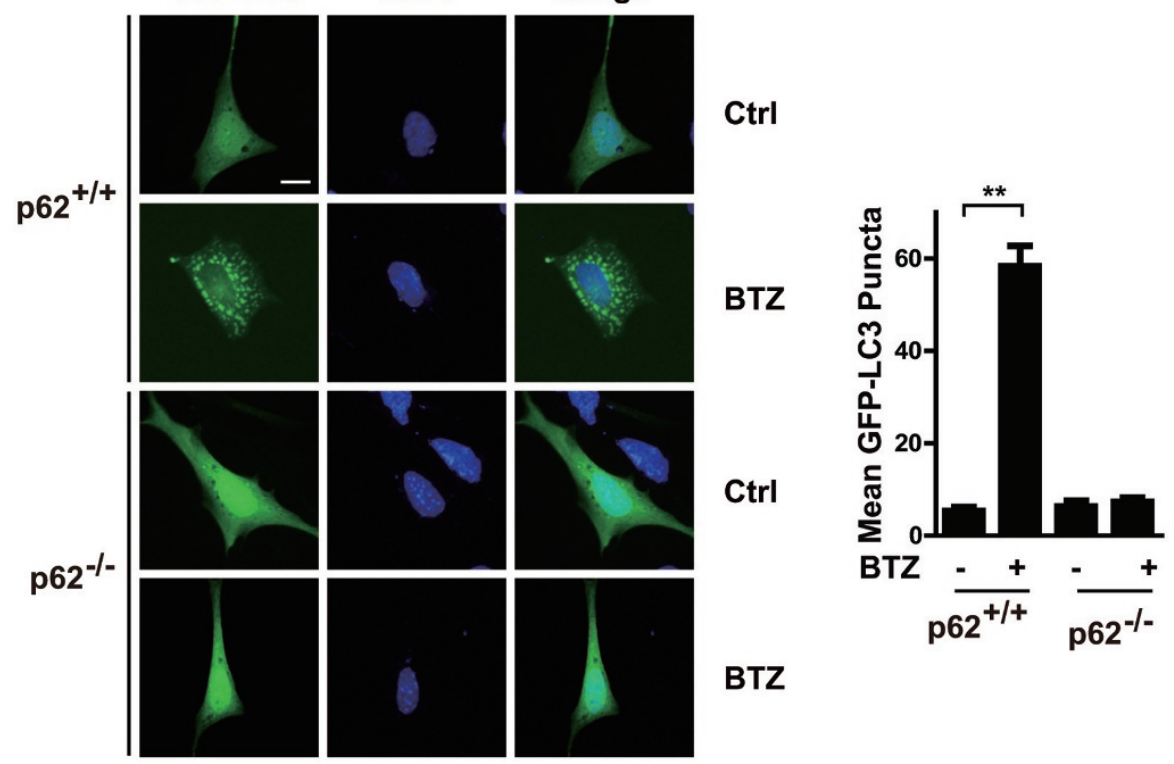

D
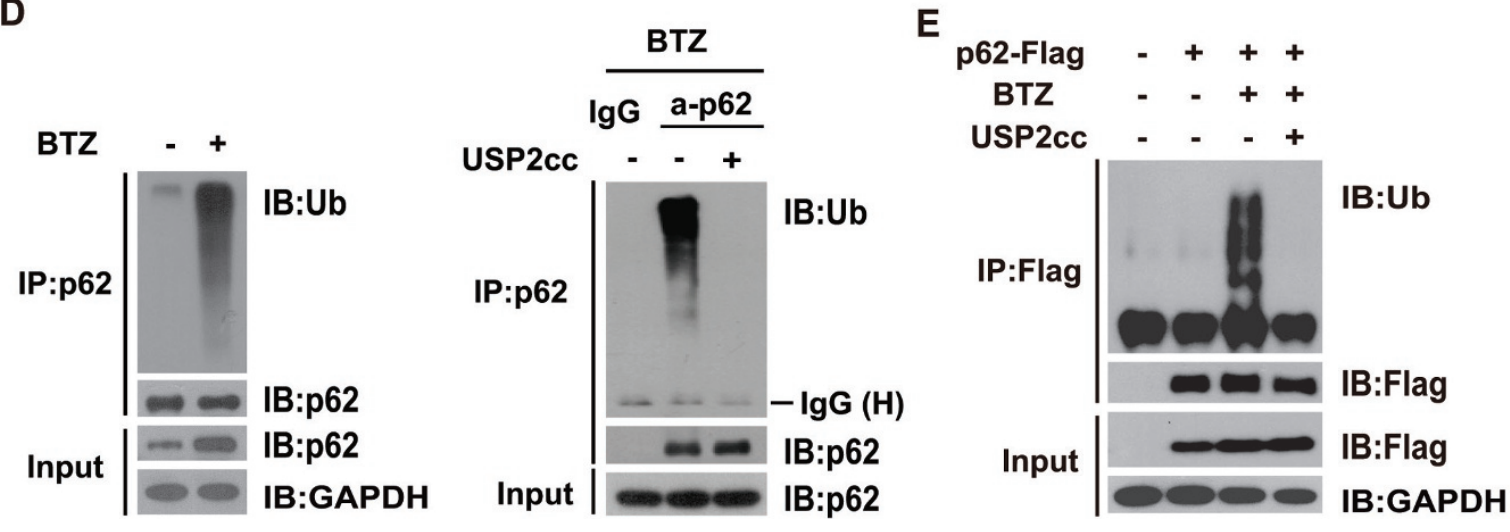

Figure 1 Autophagy receptor p62 is ubiquitylated upon autophagy activation induced by prolonged proteasome inhibition. (A) Autophagy was activated upon proteasomal inhibition in HeLa cells treated with bortezomib (BTZ, $1 \mu \mathrm{M}$ for 6 or $12 \mathrm{~h}$ ). (B) p62 was required for autophagy activated by BTZ. p62 ${ }^{-/-}$MEF cells stably expressing wild-type human p62 treated with BTZ $(1 \mu \mathrm{M}$ for $12 \mathrm{~h}$ ) or bafilomycin-A1 (BAF, $200 \mathrm{nM}$ for $8 \mathrm{~h}$ ) with the indicated combinations. Cells were lysed and analysed with indicated antibodies. The levels of lipidated LC3 were quantitated after normalization of that in each control as 1.0 . (C) p62 was required for autophagy activated by BTZ. Puncta formation by GFP-LC3 was promoted in $p 62^{+/+}$but not $p 62^{-/-}$MEF cells. Scale bar, $10 \mu \mathrm{m}$. (D, E) Endogenous or Flag-tagged p62 was ubiquitylated upon proteasome inhibition by BTZ (1 $\mu \mathrm{M}$ for 12 h) in HeLa (D) or HEK293T cells (E). Lysates were immunoprecipitated in denaturing RIPA buffer with indicated antibodies. Immunoprecipitates were treated with or without Ub-specific protease 2 (USP2cc), followed by immunoblotting analysis with indicated antibodies. 
compared to $110 \%$ increase by BTZ treatment alone (Figure 1B). More strikingly, co-treatment of BTZ and BAF led to up to $170 \%$ increase in the level of lipidated LC3, suggesting that $\mathrm{p} 62$ is required for the upregulation and faster turnover of the autophagy substrate, the lipidated LC3 (Figure 1B). As shown in Figure 1C, only in $p 62^{+/+}$but not $p 62^{-/-}$MEF cells did the BTZ treatment induce the puncta formation by GFP-LC3. Altogether, BTZ-induced proteasomal inhibition appeared to activate autophagy in a p62-dependent manner.

However, multiple previous studies have demonstrated that the C-terminal UBA domain of p62 tends to form a stable intermolecular homodimer $[35,36]$ that would spatially obstruct it from recognizing and sorting Ub conjugates for autophagic degradation. It is thus intriguing to ask how elevated p62, if indeed at self-inhibitory conformation, might contribute to autophagy activated upon BTZ-induced proteasomal inhibition.

We next investigated whether some posttranslational modifications such as ubiquitylation could modulate the ability of p62 to act as an autophagy receptor under conditions of proteasomal inhibition. To this end, both endogenous and exogenous p62 protein were immunoprecipitated from BTZ-treated HeLa or HEK293T cells in denaturing RIPA buffer, a procedure that affords efficient removal of NBR1 or Ub conjugates that might associate with p62 (Figure 1D, 1E; Supplementary information, Figure S1B and S1C). Immunoblotting (IB) analysis with anti-Ub revealed that BTZ treatment resulted in a shift of endogenous or exogenous p62 to higher molecular weight forms. These could be completely eliminated by treatment with the catalytic core of human Ub-specific protease 2 (Figure $1 \mathrm{D}$ and $1 \mathrm{E}$ ). It was clear that ubiquitylated p62 protein did accumulate following prolonged proteasome inhibition.

As shown in Supplementary information, Figure S1E, endogenous p62 protein was also found to be increas- ingly ubiquitylated in preparations of total lymphocytes (which included large amounts of tumors cells) from multiple myeloma patients, $12 \mathrm{~h}$ after intravenous administration of BTZ/Valcade. This suggested that proteasomal inhibition could indeed lead to increased ubiquitylation of $\mathrm{p} 62$ protein in vivo.

\section{Overexpression of $U b$ alone activates autophagy in a p62-dependent manner}

Interestingly, we also observed that overexpression of $\mathrm{Ub}$ led to ubiquitylation of endogenous or exogenous p62 protein in HeLa or HEK293T cells (Figure 2A, 2B; Supplementary information, Figure S1B). Together with the data that, under harsh denaturing conditions $(8 \mathrm{M}$ urea lysis buffer), exogenous p62 was also detected in the cellular polyubiquitylated conjugates recovered from cells expressing $\mathrm{His}_{6}$-tagged Ub (Supplementary information, Figure S1D), it was clear that p62 protein did undergo ubiquitylation under $\mathrm{Ub}^{+}$stress.

As the lysine-free $\mathrm{Ub}$ mutant $\left(\mathrm{Ub}_{\mathrm{K} 0}\right)$ could be conjugated to p62 as efficiently as the wild-type $\mathrm{Ub}\left(\mathrm{Ub}_{\mathrm{wT}}\right)$, it was likely that monoubiquitylation might occur dominantly on p62 (Supplementary information, Figure S1F). As $\mathrm{p} 62_{\triangle \mathrm{UBA}}$, the UBA deletion mutant of $\mathrm{p} 62$ protein, was ubiquitylated almost as efficiently as the wild-type protein, the UBA domain of p62 appeared to be not required for such ubiquitylation (Supplementary information, Figure S1G).

Surprisingly, overexpression of Ub was also found to transcriptionally upregulate $\mathrm{p} 62$; increase puncta formation by GFP-LC3; and promote lipidation of LC3, whose level could be further increased by treatment with BAF (Supplementary information, Figure S2A; Figure 2C-2E). Consistent with these diagnostic features of autophagy activation [40], we found that BTZ treatment did not cause accumulation of carbonylated proteins but instead induced their faster degradation through

Figure 2 Overexpression of Ub alone activates autophagy in a p62-dependent manner. (A, B) Endogenous or Flag-tagged p62 was ubiquitylated upon $\mathrm{Ub}^{+}$stress induced by Ub overexpression in HeLa cells (A) or HEK293T cells (B). Lysates were immuno-precipitated in denaturing RIPA buffer with indicated antibodies. Immunoprecipitates were treated with or without Ubspecific protease 2 (USP2cc), followed by immunoblotting analysis with indicated antibodies. (C) Puncta formation by GFPLC3 was promoted in HeLa cells overexpressing Ub ( 500 pmol/mg total proteins). Scale bar, $10 \mu \mathrm{m}$. (D) Autophagy might be the main route to proteolytically remove cellular oxidatively damaged (carbonylated) proteins. ATG $7^{+/+}$or $A T G 7^{-/-} \mathrm{MEF}^{-}$ cells were transfected with vector or His-Ub, cultured for $24 \mathrm{~h}$ and treated with DMSO or bafilomycin-A1 (BAF, 200 nM for 8 h). Carbonylated proteins were visualized through derivatization with DNPH, followed by IB with anti-DNP. The levels of lipidated LC3 were quantitated, after normalization of that in each control as 1.0. (E) Autophagy was activated upon Ub ${ }^{+}$stress induced by overexpression of Ub. HEK293T cells were transfected with vector or His-Ub, cultured for $24 \mathrm{~h}$ and treated with DMSO or BAF (200 nM for 8 h). Pyocyanin (PCN) was used as a positive control, and N-acetyl-L-cysteine (NAC) was used as a negative control. Carbonylated proteins were visualized through derivatization with DNPH, followed by IB with anti-DNP. $(\mathbf{F}, \mathbf{G})$ p62 was required for autophagy activated by $\mathrm{Ub}^{+}$stress. $(\mathbf{F}) p 62^{+/+}$or $p 62^{-/-} \mathrm{MEF}$ cells were subjected to $\mathrm{Ub}^{+}$stress induced by overexpression of Ub. (G) $p 62^{-/-}$MEF cells with or without re-introduction of Flag-tagged human p62. The levels of lipidated LC3 were quantitated, after normalization of the signal in each control as 1.0. 
A

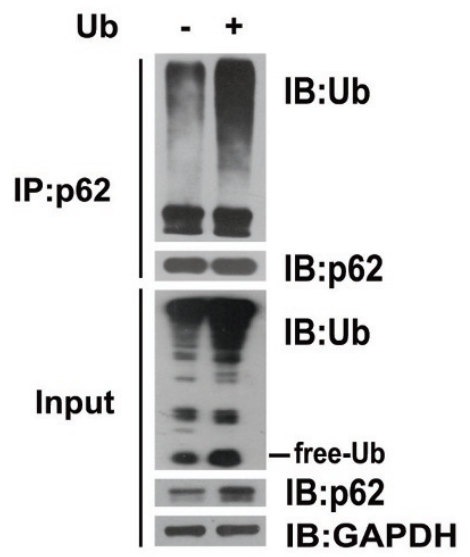

C
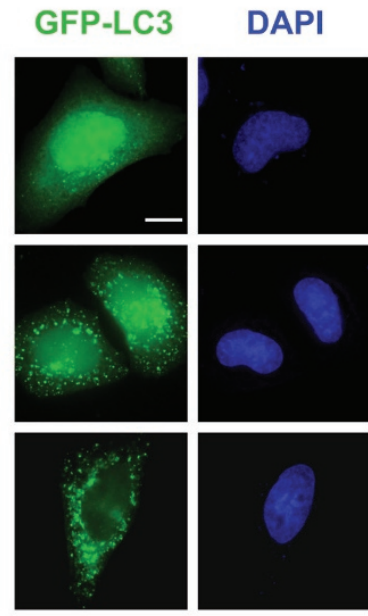

Merge

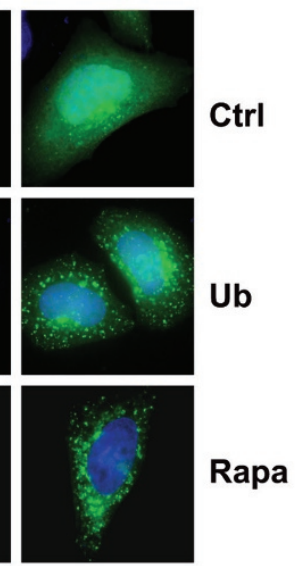

E

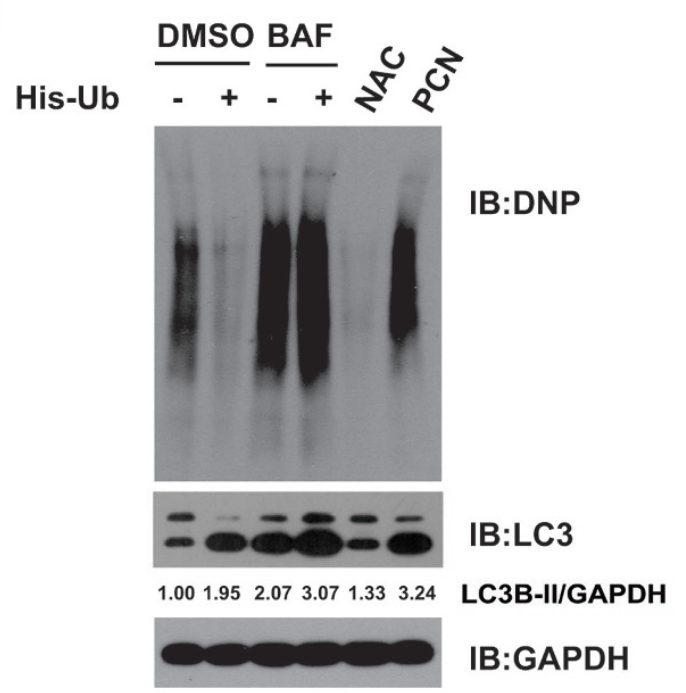

B

$\begin{array}{ccccc}\text { p62-Flag } & - & + & + & + \\ \text { Ub } & - & - & + & + \\ \text { USP2cc } & - & - & - & +\end{array}$

IP:Flag

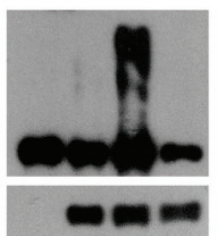

IB:Ub

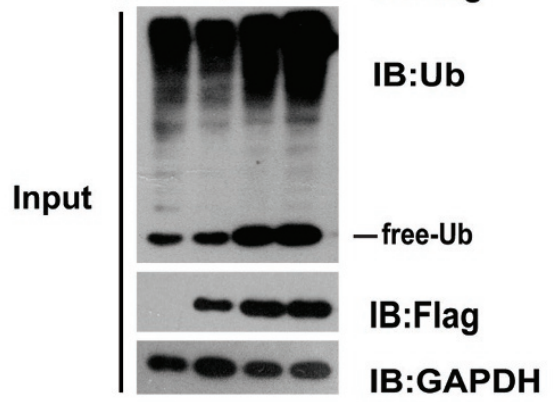

D

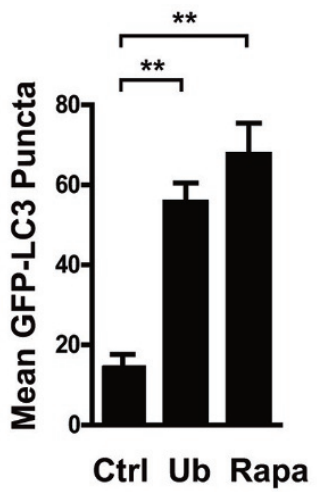

IB:DNP

IB:LC3

$\begin{array}{lll}1.00 \quad 2.36 & 0.97 \quad 0.57\end{array}$ LC3B-II/GAPGH IB:GAPDH
F

$$
\begin{aligned}
& \text { Ub } \frac{p 62^{+/+}}{-+} \frac{\mathrm{p} 62^{-/-}}{-+} \\
& 58= \\
& \text { IB:p62 } \\
& \begin{array}{llll}
1.00 & 1.60 & 0.32 & 0.20
\end{array} \\
& \text { IB:LC3 } \\
& \text { LC3B-II/GAPDH } \\
& \text { IB:GAPDH }
\end{aligned}
$$

G

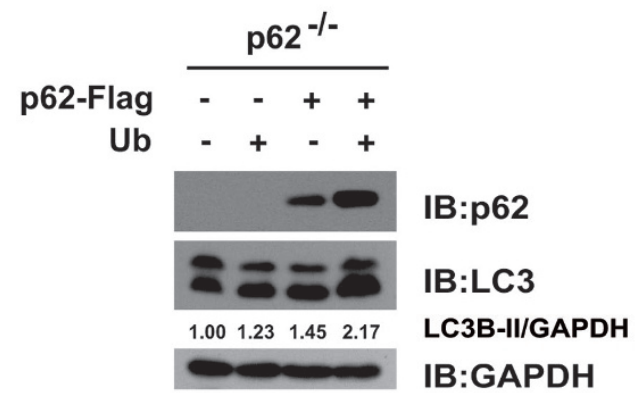


autophagy. Accordingly, treatment with BAF to inhibit autophagy did lead to stabilization of the oxidized proteins (Supplementary information, Figure S2E). We also found that degradation of cellular oxidatively damaged (carbonylated) proteins was significantly accelerated in cells overexpressing Ub. This could be blocked by BAF treatment or by genetic ablation of $A T G 7$ (Figure 2D and $2 \mathrm{E}$ ), indicating that cellular carbonylated proteins were degraded through autophagy pathway.

Furthermore, the threshold concentration for Ub overexpression to induce autophagy was found to be $\sim 500$ pmol free Ub per mg total cell lysate (Supplementary information, Figure S2F), compared to a basal level of free $\mathrm{Ub}$ of around $250 \mathrm{pmol} / \mathrm{mg}$ cell lysate [3]. This strongly suggests that a doubling of the pool of free $\mathrm{Ub}$, either untagged $\mathrm{Ub}$ or $\mathrm{Ub}$ tagged with $\mathrm{HA}$ or $\mathrm{His}_{6}$ (Supplementary information, Figure S2D), could sufficiently activate autophagy in cells. Therefore, $\mathrm{Ub}^{+}$stress induced by $\mathrm{Ub}$ overexpression (hereafter referring to at least a doubling of the free Ub pool, unless otherwise indicated) appeared to be sufficient to activate autophagy in HeLa or HEK293T cells.

We then depleted endogenous Ub by RNA interference targeting transcripts of $U b b$ in HeLa cells, using siRNAs of proven efficacy [41]. As shown in Supplementary information, Figure S2C, knocking down endogenous $\mathrm{Ub}$ appeared to downregulate LC3I as well as lipidation of LC3, suggesting an inhibitory effect of depleting the pool of free $\mathrm{Ub}$ on the basal level of autophagy. Our data also indicated that autophagy could be activated in diverse cell types by $\mathrm{Ub}$ overexpression (Figure 2; Supplementary information, Figure S2C and S2D), indicating a cell type-independent mechanism that might generally underlie the cellular response to $\mathrm{Ub}^{+}$stress resulting from $\mathrm{Ub}$ overexpression. Of note, given that overexpression of $\mathrm{Ub}$ or its mutants is commonly practiced in studies of in vivo Ub signaling, caution might be needed by those investigating functional consequences of $\mathrm{Ub}$ signaling.

We next asked whether p62 was required for autophagy activation upon $\mathrm{Ub}^{+}$stress induced by $\mathrm{Ub}$ overexpression. As shown in Figure 2F, Ub overexpression only promoted lipidation of LC3 in $p 62^{+/+}$but not $p 62^{-/-} \mathrm{MEF}$ cells. $\mathrm{Ub}^{+}$stress-activated autophagy was restored when wild-type p62 was re-introduced into $p 62^{-/-}$MEF cells (Figure $2 \mathrm{G}$ ), suggesting that p62 is critically involved in this $\mathrm{Ub}^{+}$stress-induced autophagy.

\section{Heat shock also activates autophagy through p62}

Heat shock has been long known to upregulate expression of $\mathrm{Ub}$, as well as two other E2 Ub conjugating enzymes, UBE2D2 and UBE2D3 [7, 9], thus qualifying heat shock as a $\mathrm{Ub}^{+}$stress condition. Meanwhile, there were also reports that heat shock could activate autoph- agy through mechanisms yet incompletely understood $[13,42]$. As shown in Figure $3 \mathrm{~A}$, heat shock $\left(42{ }^{\circ} \mathrm{C}, 15\right.$ or $30 \mathrm{~min}$ ) did indeed seem to increase levels of endogenous UBE2D2, UBE2D3 in HEK293FT cells. Notably, as previously reported, heat-shock treatment led to elevation in levels of poly-Ub conjugates rather than free Ub. Meanwhile, expression of endogenous p62 was also transcriptionally upregulated upon heat shock (Supplementary information, Figure S2B). As shown in Figure 3 and Supplementary information, Figure S1A, heat shock markedly increased puncta formation by GFP-LC3, and the lipidation of LC3, which could be further promoted by the treatment with BAF. These data clearly suggested that autophagy might be indeed activated in these cells upon heat shock.

Interestingly, endogenous p62 protein was significantly increased during heat shock primarily due to upregulated gene transcription (Figure 3A). Endogenous p62 was found to undergo increased ubiquitylation upon heat shock, after normalization of input amounts (Figure 3A and 3B). Furthermore, such heat-shock effects were nearly abolished when endogenous $\mathrm{Ub}$ was depleted through RNA interference targeting $U b b$ transcripts [41] (Figure 3B and data not shown). Therefore, just as with the other two $\mathrm{Ub}^{+}$stress conditions studied in this work (Figures 1-2), heat shock appeared to upregulate the expression of endogenous p62, the E2s and $\mathrm{Ub}$, thus promoting the ubiquitylation of p62 and activating autophagy through upregulation of $\mathrm{Ub}$ homeostasis.

UBE2D2/UBE2D3 interacts with p62 in vitro and in vivo

Having demonstrated that p62 was ubiquitylated upon autophagy activation induced by all the above three types of $\mathrm{Ub}^{+}$stress conditions (Figures 1-3), we next sought to identify factors that might support or mediate polyubiquitylation of p62 through the identification of p62-interacting proteins via yeast two hybrid (Y2H) screening. To this end, human p62 was employed as a bait to screen a $\mathrm{Y} 2 \mathrm{H}$ prey library containing 15000 human open reading frames for potential p62-interacting partners. Interestingly, UBE2D2 and UBE2D3, two out of the forty E2 Ub-conjugating enzymes, were found to interact with $\mathrm{p} 62$, as indicated by survival assay on yeast SD-4 (SDLeu-Trp-His-Ade) selection media as well as plate assays for $\beta$-galactosidase activity (Figure 4A). GST pull-down assays with the recombinant forms of these proteins indicated that human $\mathrm{p} 62$ protein directly interacted with UBE2D2 or UBE2D3 in vitro (Figure 4B). Co-immunoprecipitation (Co-IP) assays further indicated that p62 (both endogenous and exogenous) might indeed form a complex with UBE2D2 or UBE2D3 in vivo (Figure 4C; Supplementary information, Figure S3). This was further supported by the observation that red fluorescent pro- 
A

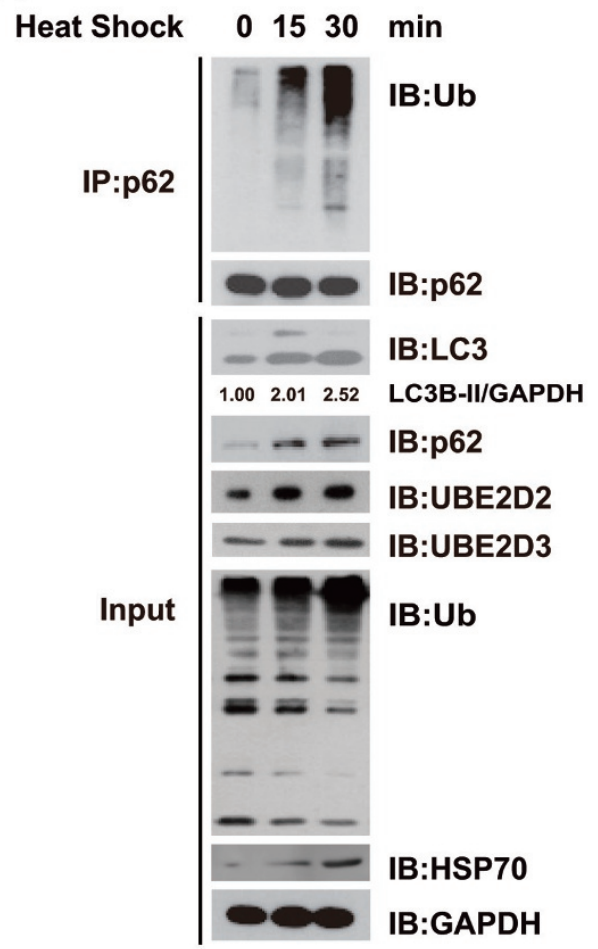

C
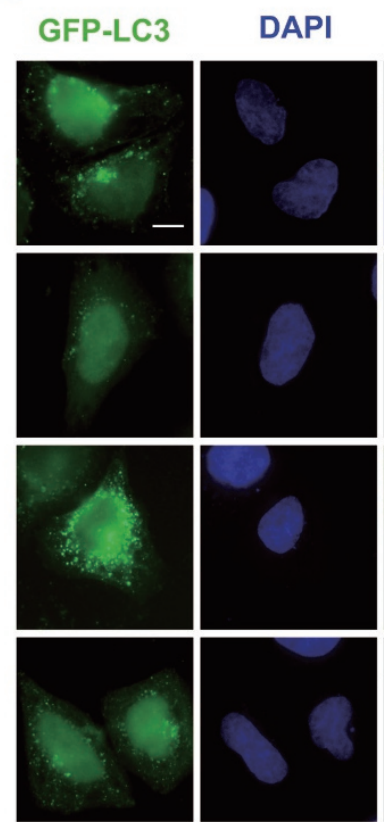

Merge

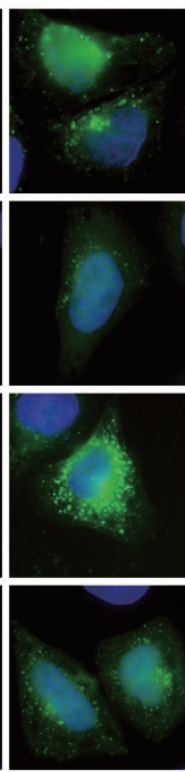

B

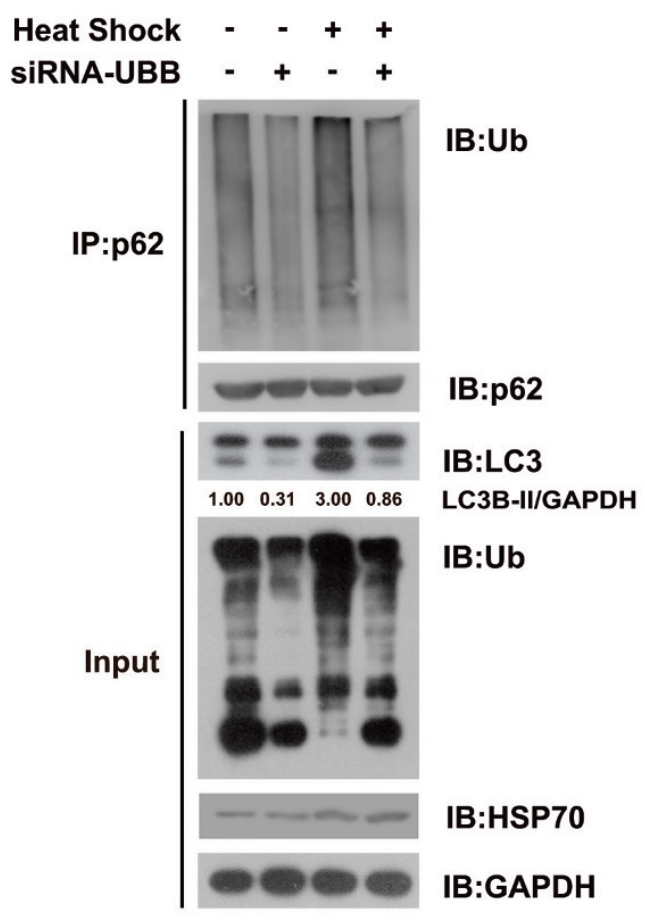

D

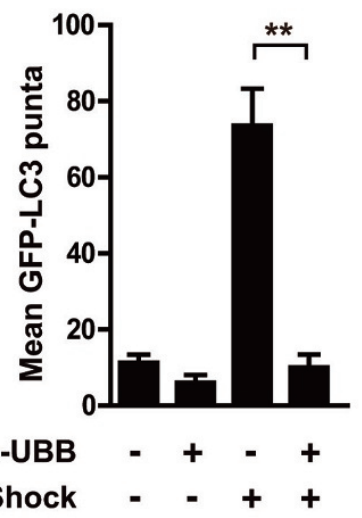

Heat Shock

E

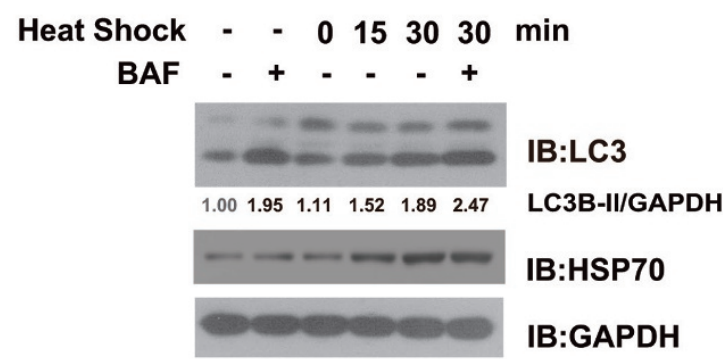

Figure 3 Heat shock also activates autophagy in dependence of p62. Endogenous human p62 underwent ubiquitylation upon autophagy activation during $\mathrm{Ub}^{+}$stress induced by heat shock. HEK293FT cells transfected with or without siRNA-UBB, were subjected to heat shock $\left(42^{\circ} \mathrm{C}, 30\right.$ min or indicated times) prior to harvest, lysed in RIPA buffer, and followed by immunoblotting analysis with indicated antibodies, HSP70 used here as an indication of heat-shock treatment (A, B, E). (E) About $36 \mathrm{~h}$ after transfection, cells were treated with DMSO or bafilomycin (BAF, $200 \mathrm{nM}, 8 \mathrm{~h}$ ). The levels of lipidated LC3 were quantitated, after normalization of that in each control as 1.0. Puncta formation by GFP-LC3 in HeLa cells in indicated groups was visualized (C) and quantitated (D). Scale bar, $10 \mu \mathrm{m}$. 
tein-tagged $\mathrm{p} 62$ protein co-localized with enhanced green fluorescent protein-tagged UBE2D2 or UBE2D3 (Figure 4D). In addition, a fragment spanning residues 294-320 of human p62 protein was mapped as the E2-interacting region (EIR) that p62 might use to directly interact with UBE2D2 or UBE2D3 in vitro (Supplementary information, Figure S4), and the deletion of EIR seemed to have no effect on p62-LC3 interaction in vitro (Supplementary information, Figure S6A).

UBE2D2-/UBE2D3-p62 interaction is critical for $\mathrm{Ub}^{+}$ stress-activated autophagy

In an in vitro ubiquitylation system, which contained $\mathrm{Mg}^{2+}$, ATP, UBA1, UBE2D2/UBE2D3 and p62 as substrate, p62 underwent UBE2D2-/UBE2D3-dependent ubiquitylation in vitro (Supplementary information, Figure S5A). Moreover, p62 $2_{\text {EIR }}$, the p62 deletion mutant that lacked the EIR, was only poorly ubiquitylated in HeLa cells overexpressing Ub, compared to wild-type p62 (Figure 4E), indicating that UBE2D2/UBE2D3 might indeed support ubiquitylation of $\mathrm{p} 62$ in vivo.

Meanwhile, as the UBA deletion mutant of p62, p62 ВА, was also efficiently ubiquitylated in vitro (Supplementary information, Figure S5B), the E2-supported p62 ubiquitylation seemed to be independent of its UBA domain, in accord with the previous in vivo data (Supplementary information, Figure S1G). This E2-dependent ubiquitylation mechanism appears to differ from the previously described monoubiquitylation of Ub-binding proteins that is mediated via UBDs and E2-conjugating enzymes [43, 44].

We next asked how E2-p62 interaction might impact on activation of autophagy upon the three types of $\mathrm{Ub}^{+}$ stress conditions. Notably, in addition to conventional LC3 lipidation assay, we employed DNP labeling coupled with anti-DNP IB analyses to assess cellular autophagic activities, with the knowledge that autophagy might be the main route to proteolytically remove cellular oxidatively damaged (carbonylated) proteins [4547] (Figure 2D, 2E; Supplementary information, Figure $\mathrm{S} 2 \mathrm{E})$. As shown in Figure $5 \mathrm{~A}$, when $\mathrm{p} 62_{\triangle \mathrm{EIR}}$ or p62 $2_{\triangle \mathrm{UBA}}$ were re-introduced into $p 62^{-1-}$ MEF cells, BTZ treatment $(1 \mu \mathrm{M}$ for $12 \mathrm{~h})$, they not only induced much less increase in lipidated LC3 compared with wild-type p62, but also resulted in more residual carbonylated proteins, which could be detected through anti-DNP assay. These data clearly suggested that both E2-dependent ubiquitylation and the intact UBA domain were required for autophagy activation upon proteasomal inhibition.

As shown in Figure 5B, carbonylated proteins were accumulated in $p 62^{-/-}$MEF cells with or without $\mathrm{Ub}$ overexpression (Lane 1 and Lane 2); however, when wild-type human p62 was re-introduced into these cells, overexpression of $\mathrm{Ub}$ led to drastic decrease in the level of residual amount of cellular carbonylated proteins together with an increase of in lipidated LC3 by $~ 110 \%$ (Lane 4). Remarkably, this was totally abolished when p62 ${ }_{\triangle E I R}$, the p62 deletion mutant that lacked the EIR, was re-introduced into $p 62^{-/-}$MEF cells (Lane 6), suggesting that E2-dependent ubiquitylation is critical for autophagic degradation of the carbonylated proteins upon $\mathrm{Ub}$ overexpression. Finally, we asked whether the E2-dependent ubiquitylation domain and the intact UBA domain were also required for autophagy activation upon heatshock treatment. Likewise, E2-p62 interaction and the intact UBA domain of p62 were also found to be required for autophagy activation induced by heat shock (Figure 5C). Taken together, this indicates p62-E2 interaction is critical for autophagy activation upon the three types of $\mathrm{Ub}^{+}$stress conditions.

\section{$U B E 2 D 2$ and UBE2D3 are the major two E2s supporting ubiquitylation of 62 in vivo}

To further investigate whether UBE2D2 and UBE2D3 might be the major two E2 Ub-conjugating ligases that support ubiquitylation of p62 upon $\mathrm{Ub}^{+}$stress, both $U B E 2 D 2$ and $U B E 2 D 3$ genes were deleted from HEK293T cells by CRISPR/Cas9 mutagenesis approach [48]. As shown in Figure 6, genetic ablation of UBE2D2 and UBE2D3 was found to almost totally abolish p62 ubiquitylation as well as autophagy activation induced by proteasomal inhibition, Ub overexpression or heat shock. Therefore, UBE2D2 and UBE2D3 are most likely the two major E2 conjugation enzymes that support p62 ubiquitylation upon the three types of $\mathrm{Ub}^{+}$stress conditions.

Ubiquitylation of p62 disrupts the dimerization of the $C$-terminal UBA domains, thus switching on its recognition of poly-Ub chains

Mass spectrometry analysis with $\mathrm{p} 62$ recovered from the in vitro ubiquitylation reaction revealed that the E2-mediated ubiquitylation occurred at least on the side chains of nine Lys residues in p62 (K157, K165, K264, K281, K295, K313, K344, K378, K420; Figure 7A; Supplementary information, Figure S5C-S5E). Four ubiquitylation sites (K157, K295, K313 and K420) were also identified from endogenous p62 recovered from HEK293T cells that overexpressed Ub (Supplementary information, Figure S5F). Interestingly, K420 was among the sites identified from both in vitro and in vivo reactions. As shown previously $[35,36]$, hydrogen bonds could be formed between the side chain of $\operatorname{Lys}_{420}\left(\mathrm{~K}_{420}\right)$ in the UBA domain of one p62 molecule and $\mathrm{Glu}_{409}\left(\mathrm{E}_{409}\right)$ 
A

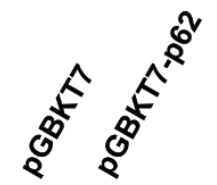

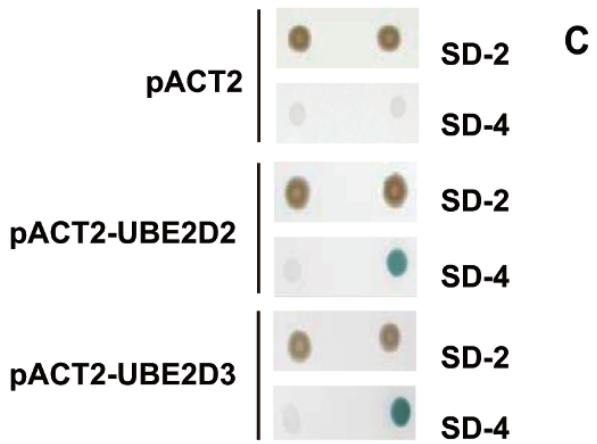

D

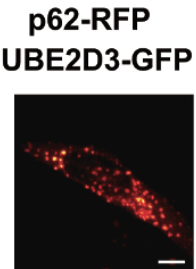

p62-RFP UBE2D2-GFP
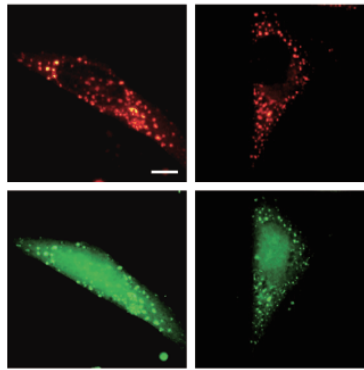

RFP
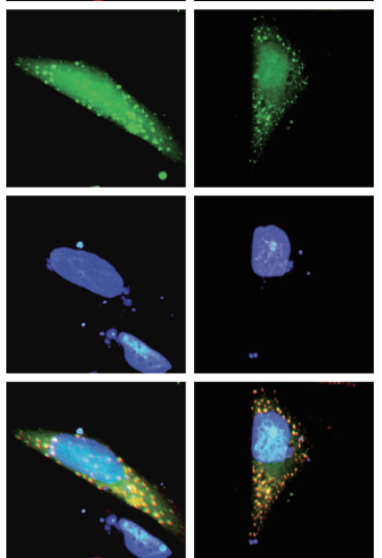

DAPI

Merge
B

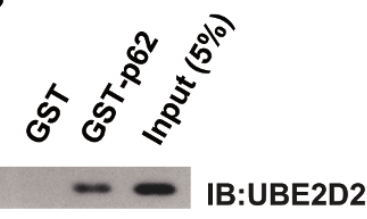

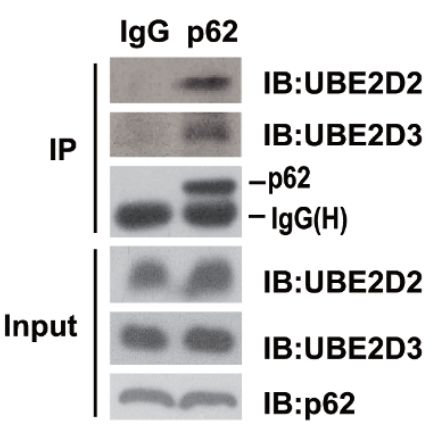

E

p62-Flag - WT $\Delta$ EIR

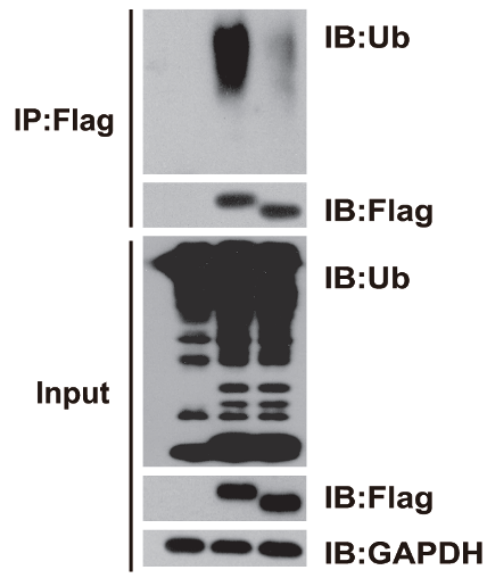

Figure 4 Human E2 conjugating enzymes, UBE2D2 or UBE2D3, interact with autophagy receptor p62 and support ubiquitylation of p62 in vitro and in vivo. (A) Human p62 interacted with UBE2D2 and UBE2D3 in yeast two hybrid assay. Co-transformation of MAV203 yeast strain with p62 and UBE2D2 or UBE2D3 supported survival of the cells on SD-4. (B) Human p62 directly interacted with UBE2D2 and UBE2D3 in GST pull-down assays carried out with purified GST-tagged p62 and Histagged UBE2D2, or His-tagged p62 and GST-tagged UBE2D3. (C) Endogenous p62 protein formed complex with UBE2D2 and UBE2D3 in vivo. IgG and p62 antibodies were used to perform immunoprecipitation in HeLa cell lysates separately, then the precipitatants were subjected to immunoblotting analysis with anti-UBE2D2 or anti-UBE2D3. (D) Human p62 co-localized with UBE2D2 or UBE2D3. HeLa cells overexpressing p62-red fluorescent protein (RFP) and UBE2D2-GFP or UBE2D3-GFP were subjected to fluorescent microscopy analysis. Scale bar, $10 \mu \mathrm{m}$. (E) Upon Ub ${ }^{+}$stress induced by overexpression of Ub, p62 underwent ubiquitylation in dependence of the interaction between p62 and UBE2D2 or UBE2D3. Human p62 interacted with UBE2D2 or UBE2D3 at E2-interacting region (EIR, spanning 294-320 residues). HeLa cells were co-transfected with His-ub and p62 ${ }_{W T}$ (wild-type human p62) or p62 ${ }_{\triangle E I R}$ (the EIR deletion mutant of p62), and lysed in RIPA buffer $36 \mathrm{~h}$ after transfection. Immunoprecipitates were then subjected to immunoblotting analysis with indicated antibodies. SD-2, deficient in Trp, Leu; SD-4, deficient in Trp, Leu, Ura and His. 
A

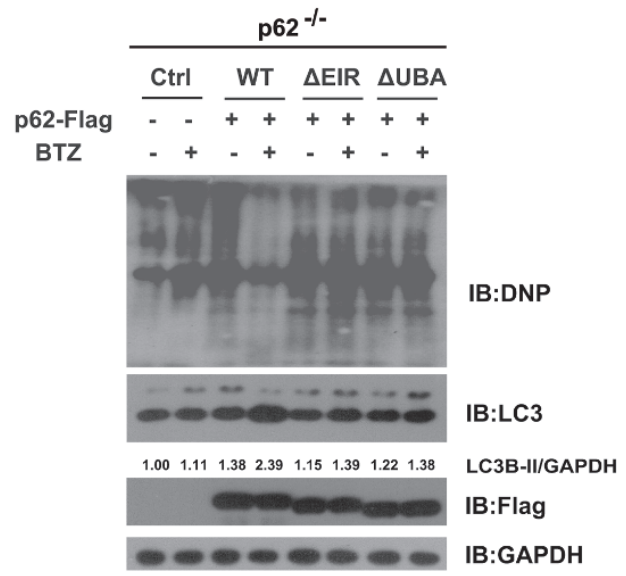

B

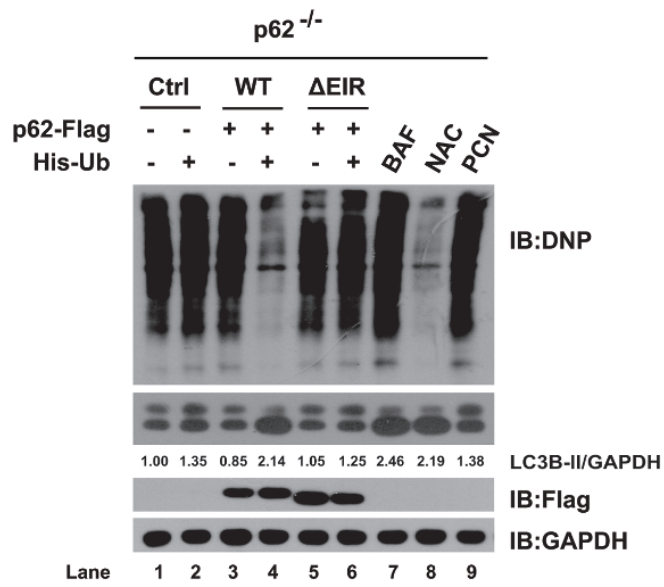

C

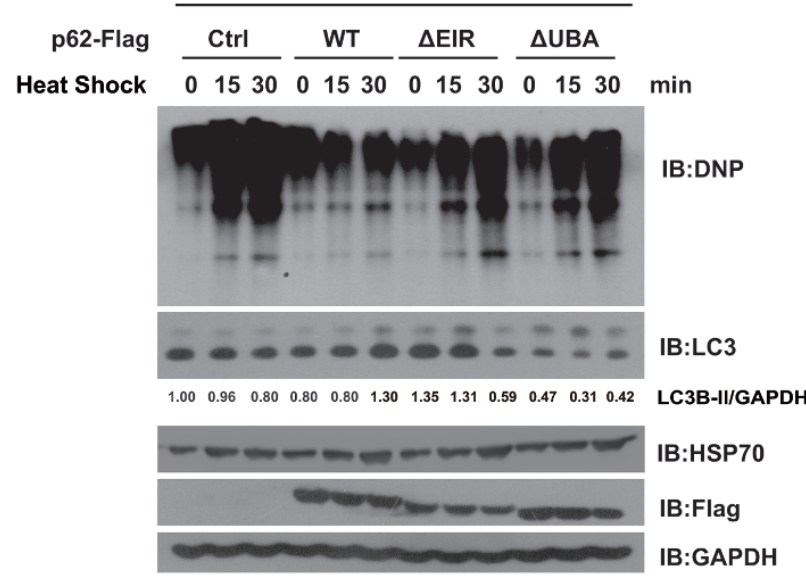

Figure 5 UBE2D2/UBE2D3-p62 interaction is critical for $\mathrm{Ub}^{+}$ stress-activated autophagy in vivo. (A-C) $p 62^{-/-} \mathrm{MEF}$ cells stably expressing p62 ${ }_{W T}$ (wild-type human p62), p62 $2_{\triangle E I R}$ (the EIR deletion mutant of p62), or p62 ${ }_{\triangle U B A}$ (the UBA deletion mutant of p62) were subjected to bortezomib treatment (BTZ, $1 \mu \mathrm{M}$ for 12 h), Ub overexpression or heat shock $\left(42{ }^{\circ} \mathrm{C}, 0,15\right.$ or $\left.30 \mathrm{~min}\right)$ before harvest. The residual cellular carbonylated proteins were visualized through derivatization with DNPH, followed by IB with anti-DNP, HSP70 used here as an indication of heat-shock treatment. The levels of lipidated LC3 were quantitated, after normalization of that in each control as 1.0 . in another p62 molecule to stabilize the intermolecular UBA dimer (Figure 7B). Although neither $\mathrm{K}_{420}$ nor $\mathrm{E}_{409}$ is directly involved into the recognition of poly-Ub chains by the UBA domain (Figure 7C), such dimerization of UBA domains would essentially prevent unmodified human $\mathrm{p} 62$ proteins from recognizing poly-Ub chains, thus locking them into an auto-inhibited state $[35,36]$. We therefore asked whether a K-to-R mutation at Lys $_{420}$ and an E-to-A mutation at $\mathrm{Glu}_{409}$ of human p62 might disrupt the formation of the intermolecular H-bond to varying extent and destabilize the UBA dimer. We found that unmodified $\mathrm{p} 62_{\mathrm{K} 420 \mathrm{R}}$ was largely defective in pulling down pre-assembled poly-Ub conjugates, in contrast to p62 $2_{\mathrm{E} 409 \mathrm{~A}}$, which pulled down conjugates much more efficiently. Not surprisingly, p62 $2_{\mathrm{K} 420 \mathrm{R} / \mathrm{E} 409 \mathrm{~A}}$ was able to pull down poly-Ub conjugates even more strongly (Supplementary information, Figure S6B). Together this data suggested that the intermolecular $\mathrm{H}$-bond between $\mathrm{K}_{420}$ and $\mathrm{E}_{409}$ might indeed constitute the major force locking the UBA dimer and preventing Ub binding. Conceivably, when p62 is ubiquitylated, particularly on K420, such intermolecular dimers could be disrupted, thus opening the "closed" conformation for recognition of poly-Ub chains.

We went on to test this hypothesis by asking whether and how E2-supported ubiquitylation of p62 might indeed affect its recognition of poly-Ub chains, an essential part of the autophagy receptor function of p62. We first reconstituted an in vitro reaction, which included ATP, HA-tagged Ub, E1 (UBA1) and E2 (UBE2D2), to efficiently assemble poly-Ub chains in the absence of E3 [49]. Poly-ubiquitylated p62 was obtained from an in vitro reaction that contained ATP, His $_{6}$-tagged $\mathrm{Ub}, \mathrm{E} 1$ (UBA1) and E2s (UBE2D2/UBE2D3), and Flag-tagged p62 or other indicated mutants. As shown in Figure 7D and 7E, unmodified p62 barely pulled down any HAtagged poly-Ub chains, suggesting that unmodified p62 might indeed have inactive status, in which the access of poly-Ub chains to UBA domain is blocked. In contrast, poly-ubiquitylated $\mathrm{p} 62, \mathrm{p} 62_{(\mathrm{Ub}) \mathrm{n}}$, could efficiently bind to, and pull down HA-tagged poly-Ub chains. In contrast, p62 $2_{9 \mathrm{KR}}$, the p62 mutant that bears K-to-R substitution at all nine ubiquitylation sites and is poorly ubiquitylated, did not pull down detectable amounts of poly-Ub chains. This suggested that UBE2D2-/UBE2D3-supported ubiquitylation of p62 could indeed switch on p62 to bind to polyubiquitylated cargoes.

We next asked whether the polymerizing status of the N-terminal PB1 domain of p62 would have any effects on the efficiency of p62 ubiquitylation or its binding to poly-Ub conjugates. It was previously reported that the K7A/D69A double mutation would disrupt the oligomerization of the PB1 [50]. As shown in Figure 7D, neither 
A

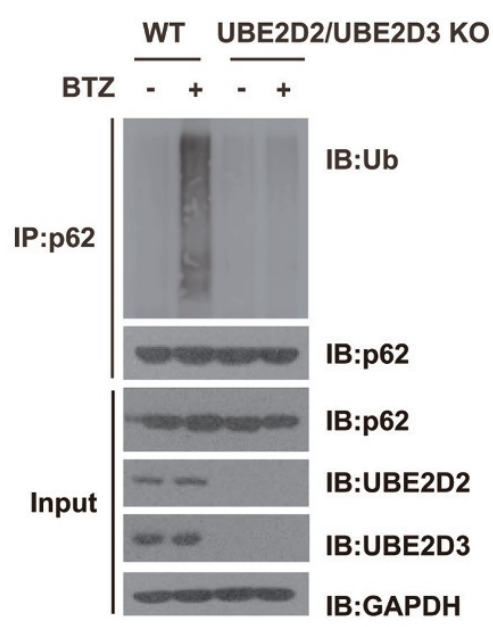

C

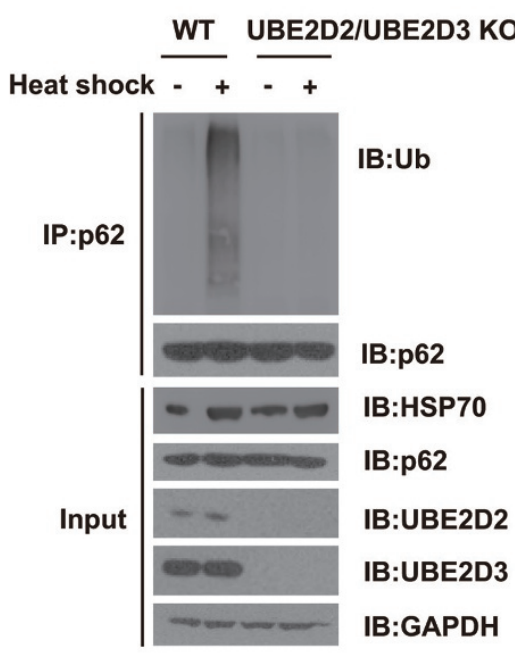

B

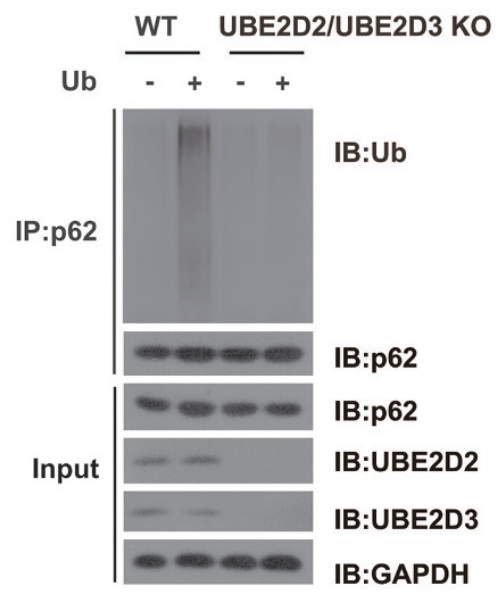

D

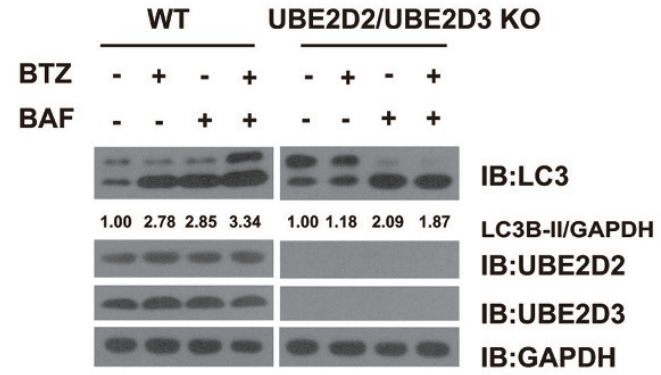

E

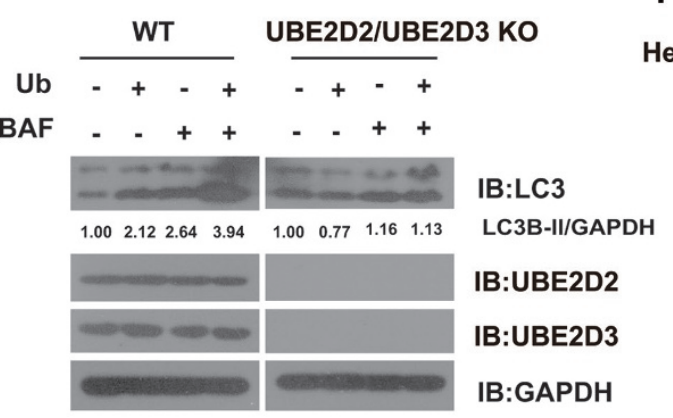


A

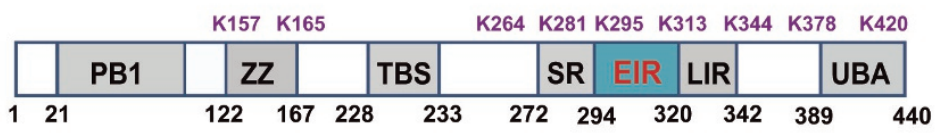

B

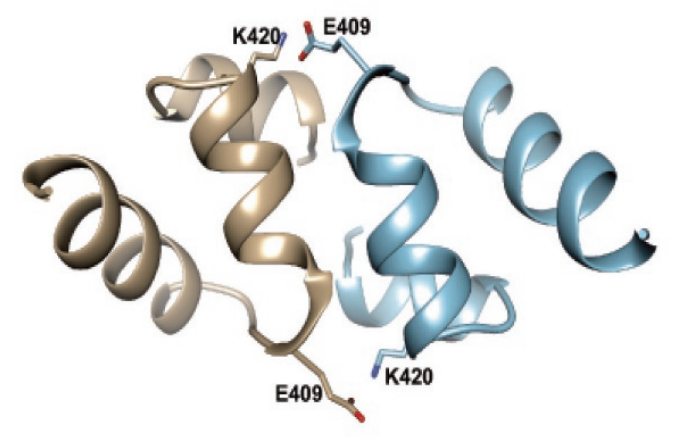

D

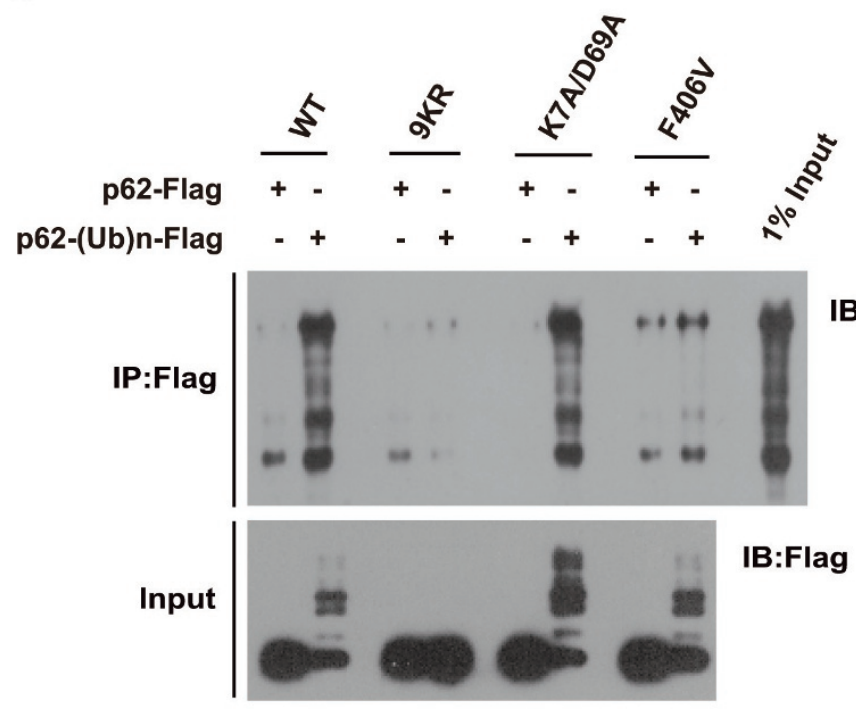

E

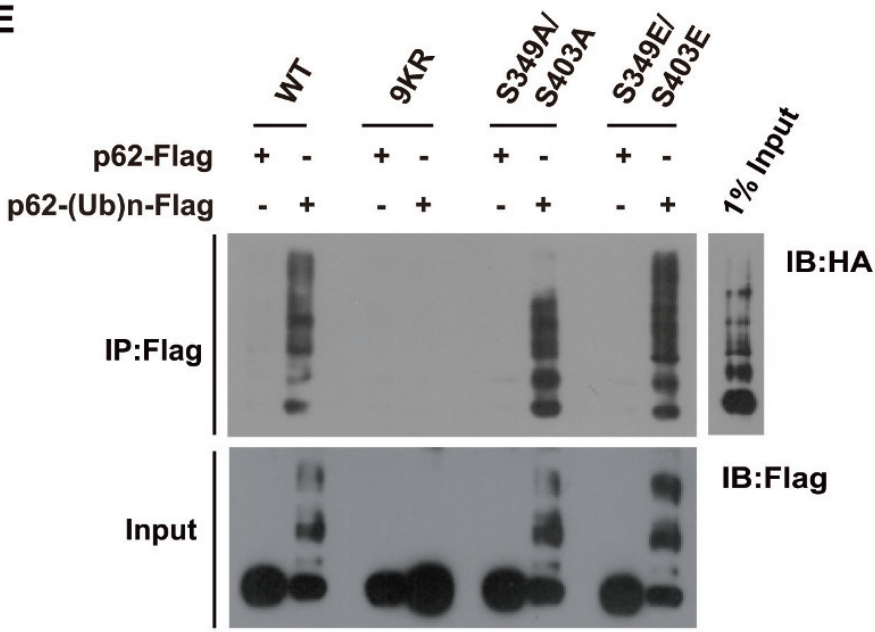

C

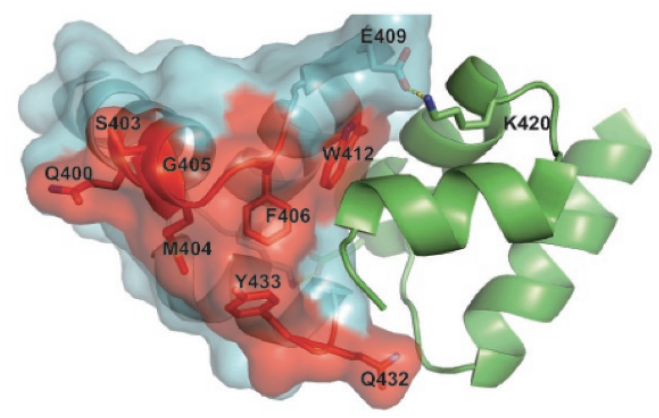

F

G HA-Ub cargoes $\quad+\quad+$
p62-Flag $\quad+-$
p62-(Ub)n-Flag $\quad+$

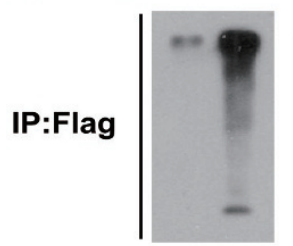

IB:HA

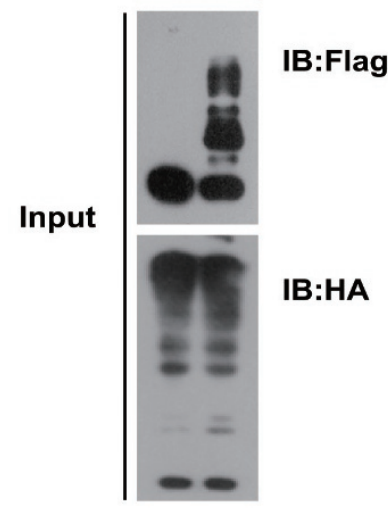


wild-type p62 nor the unmodified p62 $2_{\mathrm{K} 7 \mathrm{~A} / \mathrm{D} 69 \mathrm{~A}}$ could pull down poly-Ub chains; however, once ubiquitylated, the p62 $2_{\mathrm{K} 7 \mathrm{~A} / \mathrm{D} 69 \mathrm{~A}}$ mutant could also pull down poly-Ub chains efficiently. This strongly suggested that the oligomerization property of the PB1 domain in p62 might be not essential either for the E2-mediated ubiquitylation or recognition of poly-Ub chains (Figure 7D). Meanwhile, p62 $2_{\mathrm{F} 406 \mathrm{~V}}$, the p62 mutant that was compromised in $\mathrm{Ub}$ binding [35], could be efficiently ubiquitylated (Figure 7D), suggesting that its Ub-binding property was not required for E2-supported ubiquitylation. These data were also consistent with previous data that the UBA domain of p62 might not be essential for its ubiquitylation (Supplementary information, Figures S1G and S5B). However, unlike ubiquitylated wild-type p62 or p62 $67 \mathrm{~A} /$ ${ }_{\mathrm{D} 69 \mathrm{~A}}$, ubiquitylated $\mathrm{p} 62_{\mathrm{F} 406 \mathrm{~V}}$ could not pull down poly-Ub chains. Therefore, an intact Ub-binding property of the UBA domain appeared to be essential for the recognition of poly-Ub chains after the E2-supported ubiquitylation (Figure 7D).

Taken together, these data strongly suggest that the UBE2D2-/UBE2D3-supported ubiquitylation of p62 might disrupt dimerization of the UBA and allow efficient binding of the UBA domain to poly-Ub chains (Figure 7D), or the poly-ubiquitylated cargoes from the cell (Figure 7G). Such conformational changes are highly reminiscent of a recently demonstrated switch-on mechanism by $\mathrm{N}$-terminal arginine in $\mathrm{R}-\mathrm{BiP}$, in which the PB1 and LIR domains of p62 were exposed to potentiate the interaction with LC3 on autophagic membranes [51].

Phosphorylation at $\mathrm{Ser}^{349}$ or $\mathrm{Ser}^{403}$ is not required for E2-mediated ubiquitylation of $p 62$

Previously, phosphorylation on $\mathrm{Ser}^{403}$ in the UBA domain of p62 was found to increase its binding affinity to the ubiquitylated substrates, exemplifying the effect of a posttranslational modification on p62 to modulate its conformation and potentiate its autophagy receptor function [52, 53]. Interestingly, phosphorylation status on either $\mathrm{Ser}^{349}$ or $\mathrm{Ser}^{403}$ of p62 barely changed upon $\mathrm{Ub}^{+}$ stress induced by Ub overexpression (Figure 7F). Moreover, the full-length phosphorylation-mimicking mutant p62 $2_{\text {S349E/S403E }}$, if not ubiquitylated, could hardly pull down poly-Ub chains (Figure 7E). These results strongly suggested that phosphorylation on these sites alone might not be sufficient to turn on the recognition of poly-Ub chains by the UBA domain in full-length p62. Interestingly, ubiquitylated $\mathrm{p} 62_{\mathrm{S} 349 \mathrm{E} / \mathrm{S} 403 \mathrm{E}}$ did pull down poly-Ub chains slightly better than ubiquitylated wild-type p62 or the phosphorylation defective mutant $\mathrm{p} 62_{\mathrm{S} 349 \mathrm{~A} / \mathrm{S} 403 \mathrm{~A}}$. However, phosphorylation of $\operatorname{Ser}^{349}$ and $\mathrm{Ser}^{403}$ might not be required for E2-mediated ubiquitylation of p62 upon $\mathrm{Ub}^{+}$stress, as p62 $2_{\mathrm{S} 349 \mathrm{~A} / \mathrm{S} 403 \mathrm{~A}}$ could also undergo E2-mediated ubiquitylation efficiently clearly (Figure 7E). Yet, it remains possible that ubiquitylation and phosphorylation of p62 may work in concert to regulate autophagy under certain conditions.

Visualization of the complex formed by ubiquitylated p62 and pre-assembled poly-Ub chains

Consistent with early report that the PB1 domain in p62 could readily oligomerize [50], this domain was at least found to indeed form helical filaments under cryo-electromicroscopy [54]. A small percentage of the full-length p62 was also observed to form filaments, whose lengths were proposed to be shortened upon Ub binding. Negative stain electron microscopy (EM) analysis [55] was used to visualize and compare the complex formation by pre-assembled poly-Ub chains with p62 proteins unmodified or ubiquitylated in the presence of UBE2D2/UBE2D3. We found that incubation of ubiquitylated $\mathrm{p} 62$ protein, $\mathrm{p} 62_{(\mathrm{Ub}) \mathrm{n}}$, with pre-assembled poly-Ub chains resulted in increased formation of complexes, which were significantly larger than that for unmodified

Figure 7 Ubiquitylation of p62 switches on its recognition of poly-Ub chains, potentially through disrupting the formation of dimerization of the C-terminal UBA domains. (A) UBE2D2/UBE2D3 supported ubiquitylation of p62 in vitro on at least nine lysine residues (See Supplementary information, Figure S5 for details). All potential ubiquitylation sites in p62 were highlighted in purple. (B, C) Crystal structure of the p62 UBA dimer (PDB 3B0F) [35]. (B) Each monomer is colored in green or cyan. Lys420 and Glu409 on each monomer are shown as sticks, with the hydrogen bonds are shown as dash lines. (C) Ribbon diagram and surface representation of the p62 UBA domain dimer. Residues involved in ubiquitin binding are colored in red. (D, E) UBE2D2-/UBE2D3-supported ubiquitylation of p62 promoted the binding of the UBA domain to poly-Ub chains. Flagtagged wild-type human p62 (WT) or mutants for polyubiquitylation (9KR), oligomerization deficiency (K7A/D69A), polyubiquitin binding deficiency mutant (F406V), phosphorylation deficiency mutant (S349A/S403A) and phosphorylation-mimicking mutant (S349E/S403E) were subjected to in vitro ubiquitylation reaction, unmodified or ubiquitylated p62 proteins enriched by anti-Flag M2 beads were incubated with pre-assembled HA-tagged poly-Ub chains. (F) Phosphorylation status on either Ser349 or Ser403 of p62 barely changed upon autophagy induced by Ub overexpression in HEK293T cells. Lysates were immunoprecipitated with anti-Flag beads in denaturing RIPA buffer, and followed by IB with anti-p-p62 (S403) or anti-p-p62 (S349). (G) UBE2D2-/UBE2D3-supported ubiquitylation of p62 promoted its binding to Ub conjugates, enriched from HEK293T cells transiently expressing HA-tagged Ub. 
A p62+poly(Ub)n

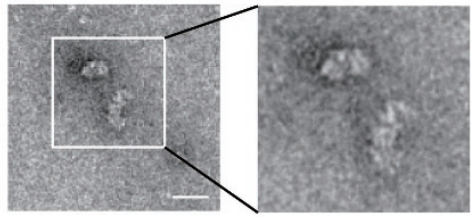

p62-(Ub)n+poly(Ub)n

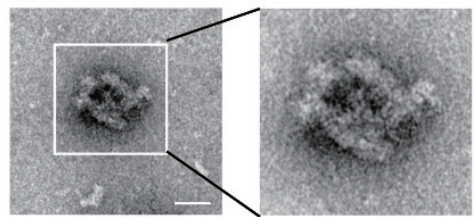

B

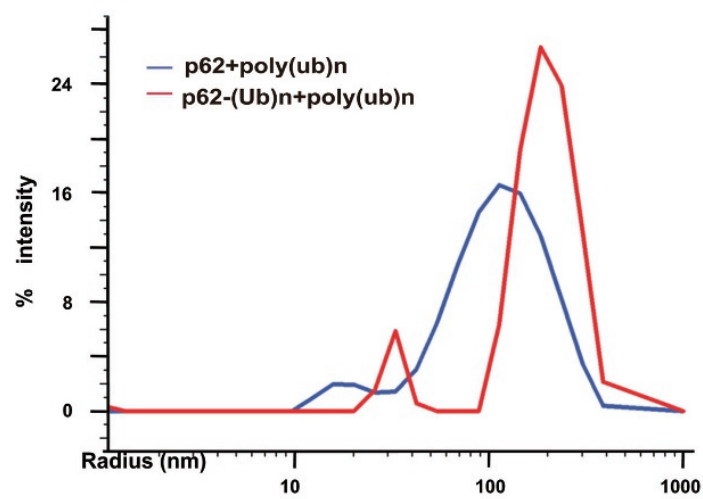

C

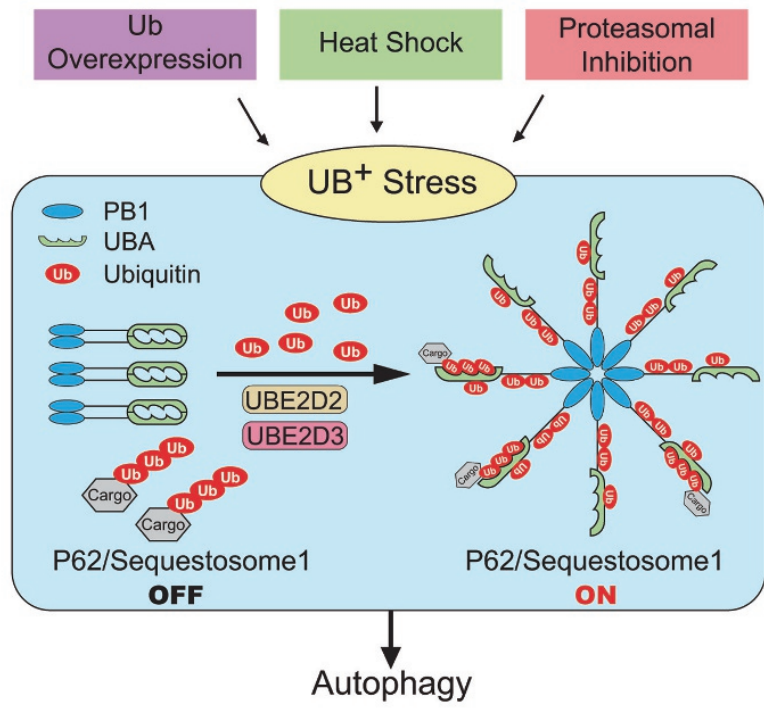

Figure 8 Visualization of the effect of p62 ubiquitylation on Ub binding. Negative stain electron microscopy (EM) (A) and dynamic light scattering (DLS) analyses (B) were performed to visualize and compare the complex formed by pre-assembled poly-Ub chains and unmodified or ubiquitylated p62 proteins. Scale bar: $50 \mathrm{~nm}$. (C) A model depicting the "sensor" role of p62 in activating autophagy upon $\mathrm{Ub}^{+}$stress induced by heat shock, Ub overexpression or proteasomal inhibition.

p62 (Figure 8A). Dynamic light scattering (DLS) analysis further indicated that $\mathrm{p} 62_{(\mathrm{Ub}) \mathrm{n}}$ formed a complex with pre-assembled poly-Ub chains with an average radius reaching $\sim 200 \mathrm{~nm}$, whereas unmodified p62 appeared to typically form aggregates of average radius around 130 $\mathrm{nm}$ (Figure 8B). These data suggested that the E2-supported ubiquitylation of $\mathrm{p} 62$ caused significant change in the conformation of $\mathrm{p} 62$. Therefore, these data directly suggest that polyubiquitylation of p62 might significantly change the conformation of $\mathrm{p} 62$ to switch on its recognition of poly-Ub chains, a modification necessarily occurring on many cargoes for selective autophagy.

In addition, p62 protein was found to undergo heavy ubiquitylation in HEK293T cells upon Salmonella infection (Supplementary information, Figure S7A), where p62 and some components of the ubiquitylation machineries were shown to be enriched locally $[56,57]$. However, there was little or no increase in p62 ubiquitylation upon autophagy activation induced by inhibition of mTOR pathway (Supplementary information, Figure S7B). This strongly suggested that E2-supported ubiquitylation of p62 might specifically underlie the activation of discrete types of autophagy.

\section{Discussion}

There is emerging evidence that Ub signaling might directly regulate the functionality of the proteasome $[58,59]$ or autophagic machineries [45]. Specifically, we reported that HACE1, a HECT domain Ankyrin repeats-containing E3 Ub ligase, mediates ubiquitylation of autophagy receptor OPTN and promotes physical interaction between autophagy receptors, OPTN and p62, to form autophagy receptor complexes, thus significantly augmenting cellular autophagic flux and "activating" autophagy [45]. Here, we first demonstrated that p62, the master autophagy regulator and receptor, specifically interacted with UBE2D2/UBE2D3 and underwent the E2-supported ubiquitylation, which destabilized dimerization of its UBA domain and activating its recognition of poly- $\mathrm{Ub}$ chains, upon $\mathrm{Ub}^{+}$stress caused by proteasomal inhibition, Ub overexpression or heat shock (Figure 8). Meanwhile, NBR1, another autophagy receptor that is structurally similar to $\mathrm{p} 62$, was also found to undergo increased ubiquitylation upon $\mathrm{Ub}^{+}$stress induced by $\mathrm{Ub}$ overexpression (Supplementary information, Figure S8A). NBR1 and p62, but not OPTN, are transcriptionally upregulated upon $\mathrm{Ub}^{+}$stress induced by $\mathrm{Ub}$ overexpression (Supplementary information, Figure S2A). Unlike OPTN, NBR1 could also interact with UBE2D2 or UBE2D3, and undergo E2-supported ubiquitylation (Supplementary information, Figure S8B-S8D), suggesting that a similar mechanism might also activate NBR1 for recognition of poly-Ub chains upon $\mathrm{Ub}^{+}$stress. This 
has added yet another dimension to the puzzle of why such two highly similar autophagy receptors exist but do not compensate for each other when one of them is lost. It is interesting to note that our findings have delineated a novel mechanism through which Ub signaling directly regulates the functionality of the autophagy receptors p62 and probably also NBR1, the key components of the proteolytic machineries, and so controls cellular autophagy.

In summary, as Ub stress and fluctuations in autophagic activity critically feature and underlie cellular responses to a wide range of stimuli including heat shock, microbe infection, ageing or drug treatment (e.g., BTZ) etc., delineation of the mechanism and regulatory roles of p62 in sensing Ub stress to control autophagy could not only bring mechanistic understanding of the related fundamental processes, but also open a new avenue to develop better therapeutics targeting autophagy-related human disorders. Notably, the findings we report here should also alert researchers of a need to be cautions when using $\mathrm{Ub}$ overexpression to study $\mathrm{Ub}$ signaling in vivo.

Recently, tripartite motif-containing protein 21 (TRIM21), a Ub E3 ligase, was reported to ubiquitylate Lys (K7) in the N-terminal PB1 domain of p62. Such ubiquitylation was found to disrupt PB1-mediated oligomerization of p62 and suppress its sequestration of specific proteins [60]. Work by Pan et al. [60] is thus significantly different from our study in many aspects. First, K7 is obviously not among the nine ubiquitylation sites that we identified from p62 ubiquitylated in in vitro reaction or those recovered from cells treated with BTZ. Moreover, TRIM21-mediated ubiquitylation of p62 was reported to depend on UBE2D2/UBE2D3, exactly the same E2 enzymes that we have demonstrated to be sufficient to mediate ubiquitylation of p62, e.g., that on K420, and activate autophagy receptor function of $\mathrm{p} 62$, without involving extra E3 Ub ligase. Finally, as oligomerization of p62 through its $\mathrm{N}$-terminal $\mathrm{PB} 1$ domain is required for p62-mediated autophagy [34, 61], TRIM21-conjugated poly-Ub chains on $\mathrm{p} 62$ should inevitably suppress the autophagy receptor function of $\mathrm{p} 62$, which is totally different from the activating role that we found for UBE2D2-/ UBE2D3-dependent ubiquitylation. Therefore, as both p62 and its ubiquitylation were required for autophagy activated by $\mathrm{Ub}^{+}$stress, TRIM21-mediated $\mathrm{p} 62$ ubiquitylation, if it does occur, might represent a mechanism for regulating p62 function in conditions other than $\mathrm{Ub}^{+}$ stress.

\section{Materials and Methods}

\section{Reagents and plasmid construction}

See Supplementary information, Data S1 and Table S1 for de- tails.

\section{Yeast two hybrid screen}

$\mathrm{Y} 2 \mathrm{H}$ screening was carried out to screen for proteins that may support ubiquitylation of p62, using human p62 as bait as previously described [45].

\section{GST pull-down, IP and IB}

GST pull-down, IP and IB were performed as described [62, 63]. Endogenous p62 was recovered through IP using anti-p62, followed by IB with the indicated antibodies. When UBE2D2 or UBE2D3 was identified as a p62-interacting protein, GST pulldown and Co-IP was carried out to confirm their interactions in vitro and in vivo. See Supplementary information, Data S1.

\section{In vitro and in vivo ubiquitylation assays}

In vitro ubiquitylation assays were performed as previously described $[49,64]$. IP with anti-p62 or indicated antibodies, followed by anti-Ub IB, was performed in denaturing RIPA buffer, with extensive washing to check the ubiquitylation status of endogenous or tagged p62 protein. See Supplementary information, Data S1.

\section{Mass spectra and mutagenesis analyses}

Mass spectra (MS) and mutagenesis analyses, were performed to determine the ubiquitylation sites on p62. Samples for MS analysis were prepared using the same in vitro ubiquitylation protocols mentioned above except that the samples were blocked with 2-chloroacetamide (Sigma) for $30 \mathrm{~min}$ at $30{ }^{\circ} \mathrm{C}$ before loading to the gel [65]. The extracts were then desalted and concentrated using StageTip [66] and the eluted peptides were subjected to MS analysis.

\section{Autophagy assay and detection of oxidatively damaged pro-} teins

Formation of GFP-LC3 puncta and the level of lipidated LC3 were assayed following the standard guidelines [40], whereas levels of residual cellular oxidatively damaged (carbonylated) proteins were determined with anti-DNP assay described before [45].

Construction of $U B E 2 D 2^{-/-}$and $U B E 2 D 3^{-/}$cell lines of HEK293T

$U B E 2 D 2^{-/-}$and $U B E 2 D 3^{-/-}$cell lines were constructed as described before [48]. A 20-bp guide sequence (5'-CCATTGGCAAGCTACAATAA- $3^{\prime}$ ) targeting DNA within the third exon of UBE2D2 and a 20-bp guide sequence (5'-AGAATACACCGCCTTGATAT-3') targeting DNA within the third exon of UBE2D3 were selected from a database of predicted high specificity protospacer adjacent motif target sites (http://crispr.mit.edu). Two complementary oligos containing the UBE2D2/UBE2D3 sequence and $B s m$ BI (NEB) ligation adapters were synthesized. The annealed oligo was ligated into the Bsm BI-digested lentiCRISPRv2 vector. The sequence of the construct was verified by DNA sequencing. HEK293T cells were seeded into 6-well plates to $90 \%$ confluence. The lentiCRISPRv2 or lentiCRISPRv2-gRNA construct was introduced into cells by transfection using Lipofectamin 2000 (Invitrogen) for $24 \mathrm{~h}$, followed by $10 \mu \mathrm{g} / \mathrm{ml}$ puromycin (Sigma-Aldrich) treatment for $36 \mathrm{~h}$ to maximally eliminate the untransfected cells. Then the surviving cells were diluted and grown into single colonies about 1 week before further gone into screen for positive 
clones.

\section{Negative EM and dynamic light scattering analyses}

Negative stain EM and DLS analyses [55] were employed to visualize and compare the complex formation by pre-assembled poly-Ub chains with $\mathrm{p} 62$ proteins unmodified or ubiquitylated in the presence of UBE2D2/UBE2D3.

Full methods and any associated references are available in Supplementary information, Data S1.

\section{Acknowledgments}

We thank Dr Wei Bian and her team in cell biology core facility of SIBCB for their excellent support in imaging. We particularly thank Dr Ron Kopito from Stanford University and Dr Yi-fan Cheng from the University of San Francisco for helpful discussions. We also thank Dr Yalan Wu and all other members of the $\mathrm{Hu}$ lab for support. We are also thankful to Dr Yefei Li for assistance with illustrations. This work was supported by the Strategic Priority Research Program of the Chinese Academy of Sciences (XDB19000000), the National Natural Science Foundation of China (31270828, 31070678 and 81525019, an award for national outstanding young scientist) and the Ministry of Science and Technology of China (2012CB910800, 2013CB910900 to RH). RH was also supported by Shanghai Institute of Organic Chemistry, CAS; and this work was also supported by the Instrument Developing Project of the Chinese Academy of Sciences (YZ201339) and by NIH grant GM095526 (to DF) and LOEWE grant Ub-Net Frankfurt, and the European Research Council/ERC grant agreement no. 250241-LineUb (to ID).

\section{Author Contributions}

$\mathrm{RH}$ and DF conceived and supervised the study. ID advised on the study. PH, JY, QY, GL, MY and BL did the experiments; RH, DF, ID, PH, YH and JY analyzed the data; RH, DF, ID, PH and JY wrote the manuscript. All authors read and approved the final manuscript.

\section{Competing Financial Interests}

The authors declare no competing financial interests.

\section{References}

1 Hershko A, Ciechanover A, Varshavsky A. Basic medical research award. The ubiquitin system. Nat Med 2000; 6:10731081.

2 Finley D, Ciechanover A, Varshavsky A. Ubiquitin as a central cellular regulator. Cell 2004; 116:S29-S32, 2p following S32.

3 Kaiser SE, Riley BE, Shaler TA, et al. Protein standard absolute quantification (PSAQ) method for the measurement of cellular ubiquitin pools. Nat Methods 2011; 8:691-696.

4 Grabbe C, Husnjak K, Dikic I. The spatial and temporal organization of ubiquitin networks. Nat Rev Mol Cell Biol 2011; 12:295-307.

5 Hanna J, Meides A, Zhang DP, Finley D. A ubiquitin stress response induces altered proteasome composition. Cell 2007; 129:747-759.
6 Lee BH, Lu Y, Prado MA, et al. USP14 deubiquitinates proteasome-bound substrates that are ubiquitinated at multiple sites. Nature 2016; 532:398-401.

7 Bond U, Schlesinger MJ. Ubiquitin is a heat shock protein in chicken embryo fibroblasts. Mol Cell Biol 1985; 5:949-956.

8 Hallengren J, Chen PC, Wilson SM. Neuronal ubiquitin homeostasis. Cell Biochem Biophys 2013; 67:67-73.

9 Seufert W, Jentsch S. Ubiquitin-conjugating enzymes UBC4 and UBC5 mediate selective degradation of short-lived and abnormal proteins. EMBO J 1990; 9:543-550.

10 Koyano F, Okatsu K, Kosako H, et al. Ubiquitin is phosphorylated by PINK1 to activate parkin. Nature 2014; 510:162166.

11 Sarraf SA, Raman M, Guarani-Pereira V, et al. Landscape of the PARKIN-dependent ubiquitylome in response to mitochondrial depolarization. Nature 2013; 496:372-376.

12 Wauer T, Swatek KN, Wagstaff JL, et al. Ubiquitin Ser65 phosphorylation affects ubiquitin structure, chain assembly and hydrolysis. EMBO J 2015; 34:307-325.

13 Gropper R, Brandt RA, Elias S, et al. The ubiquitin-activating enzyme, E1, is required for stress-induced lysosomal degradation of cellular proteins. J Biol Chem 1991; 266:3602-3610.

14 Pan JX, Short SR, Goff SA, Dice JF. Ubiquitin pools, ubiquitin mRNA levels, and ubiquitin-mediated proteolysis in aging human fibroblasts. Exp Gerontol 1993; 28:39-49.

15 Yu T, Tao Y, Yang M, et al. Profiling human protein degradome delineates cellular responses to proteasomal inhibition and reveals a feedback mechanism in regulating proteasome homeostasis. Cell Res 2014; 24:1214-1230.

16 Chen Y, Piper PW. Consequences of the overexpression of ubiquitin in yeast: elevated tolerances of osmostress, ethanol and canavanine, yet reduced tolerances of cadmium, arsenite and paromomycin. Biochim Biophys Acta 1995; 1268:59-64.

17 Hoe N, Huang CM, Landis G, et al. Ubiquitin over-expression phenotypes and ubiquitin gene molecular misreading during aging in Drosophila melanogaster. Aging (Albany NY) 2011; 3:237-261.

18 Finch JS, St John T, Krieg P, et al. Overexpression of three ubiquitin genes in mouse epidermal tumors is associated with enhanced cellular proliferation and stress. Cell Growth Differ 1992; 3:269-278.

19 Tian Y, Ding W, Wang Y, et al. Ubiquitin B in cervical cancer: critical for the maintenance of cancer stem-like cell characters. PLoS One 2013; 8: 84457.

20 Finley D, Bartel B, Varshavsky A. The tails of ubiquitin precursors are ribosomal proteins whose fusion to ubiquitin facilitates ribosome biogenesis. Nature 1989; 338:394-401.

21 Hanna J, Leggett DS, Finley D. Ubiquitin depletion as a key mediator of toxicity by translational inhibitors. Mol Cell Biol 2003; 23:9251-9261.

22 Ryu KY, Garza JC, Lu XY, Barsh GS, Kopito RR. Hypothalamic neurodegeneration and adult-onset obesity in mice lacking the Ubb polyubiquitin gene. Proc Natl Acad Sci USA 2008; 105:4016-4021.

23 Ryu KY, Sinnar SA, Reinholdt LG, et al. The mouse polyubiquitin gene $U b b$ is essential for meiotic progression. Mol Cell Biol 2008; 28:1136-1146.

24 Setsuie R, Wada K. The functions of UCH-L1 and its relation to neurodegenerative diseases. Neurochem Int 2007; 51:105- 
111.

25 Anderson C, Crimmins S, Wilson JA, Korbel GA, Ploegh HL, Wilson SM. Loss of Usp14 results in reduced levels of ubiquitin in ataxia mice. J Neurochem 2005; 95:724-731.

26 Wilson SM, Bhattacharyya B, Rachel RA, et al. Synaptic defects in ataxia mice result from a mutation in Usp14, encoding a ubiquitin-specific protease. Nat Genet 2002; 32:420425.

27 Ryu KY, Maehr R, Gilchrist CA, et al. The mouse polyubiquitin gene $U b C$ is essential for fetal liver development, cell-cycle progression and stress tolerance. EMBO J 2007; 26:26932706.

28 Klionsky DJ, Schulman BA. Dynamic regulation of macroautophagy by distinctive ubiquitin-like proteins. Nat Struct Mol Biol 2014; 21:336-345.

29 Ohsumi Y. Historical landmarks of autophagy research. Cell Res 2014; 24:9-23.

30 Rogov V, Dotsch V, Johansen T, Kirkin V. Interactions between autophagy receptors and ubiquitin-like proteins form the molecular basis for selective autophagy. Mol Cell 2014; 53:167-178.

31 Kabeya Y, Mizushima N, Ueno T, et al. LC3, a mammalian homologue of yeast Apg8p, is localized in autophagosome membranes after processing. EMBO J 2000; 19:5720-5728.

$32 \mathrm{Lu} \mathrm{K}$, Psakhye I, Jentsch S. Autophagic clearance of polyQ proteins mediated by ubiquitin-Atg 8 adaptors of the conserved CUET protein family. Cell 2014; 158:549-563.

33 Kirkin V, Lamark T, Johansen T, Dikic I. NBR1 cooperates with p62 in selective autophagy of ubiquitinated targets. $A u$ tophagy 2009; 5:732-733.

34 Johansen T, Lamark T. Selective autophagy mediated by autophagic adapter proteins. Autophagy 2011; 7:279-296.

35 Isogai $\mathrm{S}$, Morimoto $\mathrm{D}$, Arita $\mathrm{K}$, et al. Crystal structure of the ubiquitin-associated (UBA) domain of p62 and its interaction with ubiquitin. J Biol Chem 2011; 286:31864-31874.

36 Long J, Garner TP, Pandya MJ, et al. Dimerisation of the UBA domain of p62 inhibits ubiquitin binding and regulates NF-kappaB signalling. J Mol Biol 2010; 396:178-194.

37 Komatsu M, Kurokawa H, Waguri S, et al. The selective autophagy substrate p62 activates the stress responsive transcription factor Nrf2 through inactivation of Keap1. Nat Cell Biol 2010; 12:213-223.

38 Jain A, Lamark T, Sjottem E, et al. p62/SQSTM1 is a target gene for transcription factor NRF2 and creates a positive feedback loop by inducing antioxidant response element-driven gene transcription. J Biol Chem 2010; 285:22576-22591.

39 Vercauteren D, Deschout H, Remaut K, et al. Dynamic colocalization microscopy to characterize intracellular trafficking of nanomedicines. ACS Nano 2011; 5:7874-7884.

40 Klionsky DJ, Abdelmohsen K, Abe A, et al. Guidelines for the use and interpretation of assays for monitoring autophagy (3rd edition). Autophagy 2016; 12:1-222.

41 Wu P, Tian Y, Chen G, et al. Ubiquitin B: an essential mediator of trichostatin A-induced tumor-selective killing in human cancer cells. Cell Death Differ 2010; 17:109-118.

42 Komata T, Kanzawa T, Nashimoto T, et al. Mild heat shock induces autophagic growth arrest, but not apoptosis in U251MG and U87-MG human malignant glioma cells. J Neurooncol 2004; 68:101-111.
43 Hoeller D, Hecker CM, Wagner S, Rogov V, Dotsch V, Dikic I. E3-independent monoubiquitination of ubiquitin-binding proteins. Mol Cell 2007; 26:891-898.

44 Hochstrasser M. Ubiquitin ligation without a ligase. Dev Cell 2007; 13:4-6.

45 Liu Z, Chen P, Gao H, et al. Ubiquitylation of autophagy receptor optineurin by HACE1 activates selective autophagy for tumor suppression. Cancer Cell 2014; 26:106-120.

46 Simonsen A, Cumming RC, Brech A, Isakson P, Schubert DR, Finley KD. Promoting basal levels of autophagy in the nervous system enhances longevity and oxidant resistance in adult Drosophila. Autophagy 2008; 4:176-184.

47 Rubinsztein DC, Marino G, Kroemer G. Autophagy and aging. Cell 2011; 146:682-695.

48 Cong L, Ran FA, Cox D, et al. Multiplex genome engineering using CRISPR/Cas systems. Science 2013; 339:819-823.

49 Li W, Tu D, Li L, et al. Mechanistic insights into active site-associated polyubiquitination by the ubiquitin-conjugating enzyme Ube2g2. Proc Natl Acad Sci USA 2009; 106:3722-3727.

50 Lamark T, Perander M, Outzen $\mathrm{H}$, et al. Interaction codes within the family of mammalian Phox and Bem 1p domain-containing proteins. J Biol Chem 2003; 278:3456834581.

51 Cha-Molstad H, Sung KS, Hwang J, et al. Amino-terminal arginylation targets endoplasmic reticulum chaperone BiP for autophagy through p62 binding. Nat Cell Biol 2015; 17:917929.

52 Matsumoto G, Wada K, Okuno M, Kurosawa M, Nukina N. Serine 403 phosphorylation of p62/SQSTM1 regulates selective autophagic clearance of ubiquitinated proteins. Mol Cell 2011; 44:279-289.

53 Lim J, Lachenmayer ML, Wu S, et al. Proteotoxic stress induces phosphorylation of $\mathrm{p} 62 / \mathrm{SQSTM} 1$ by ULK1 to regulate selective autophagic clearance of protein aggregates. PLoS Genet 2015; 11:e1004987.

54 Ciuffa R, Lamark T, Tarafder AK, et al. The selective autophagy receptor $\mathrm{p} 62$ forms a flexible filamentous helical scaffold. Cell Rep 2015; 11:748-758.

55 Booth DS, Avila-Sakar A, Cheng Y. Visualizing proteins and macromolecular complexes by negative stain EM: from grid preparation to image acquisition. J Vis Exp 2011 Dec 22. doi:10.3791/3227

56 van Wijk SJ, Fiskin E, Putyrski M, et al. Fluorescence-based sensors to monitor localization and functions of linear and K63-linked ubiquitin chains in cells. Mol Cell 2012; 47:797809.

57 Zheng YT, Shahnazari S, Brech A, Lamark T, Johansen T, Brumell JH. The adaptor protein p62/SQSTM1 targets invading bacteria to the autophagy pathway. J Immunol 2009; 183:5909-5916.

58 Besche HC, Sha Z, Kukushkin NV, et al. Autoubiquitination of the 26S proteasome on Rpn13 regulates breakdown of ubiquitin conjugates. EMBO J 2014; 33:1159-1176.

59 Cohen-Kaplan V, Livneh I, Avni N, et al. p62- and ubiquitin-dependent stress-induced autophagy of the mammalian 26S proteasome. Proc Natl Acad Sci USA 2016; 113:E7490-E7499.

60 Pan JA, Sun Y, Jiang YP, et al. TRIM21 ubiquitylates SQSTM1/p62 and suppresses protein sequestration to regulate 
redox homeostasis. Mol Cell 2016; 62:149-151.

61 Bjorkoy G, Lamark T, Brech A, et al. p62/SQSTM1 forms protein aggregates degraded by autophagy and has a protective effect on huntingtin-induced cell death. J Cell Biol 2005; 171:603-614

62 Shen J, Sheng X, Chang Z, et al. Iron metabolism regulates p53 signaling through direct heme-p53 interaction and modulation of p53 localization, stability, and function. Cell Rep 2014; 7:180-193.

63 Inuzuka H, Gao D, Finley LW, et al. Acetylation-dependent regulation of Skp2 function. Cell 2012; 150:179-193.
64 Ravid T, Hochstrasser M. Autoregulation of an E2 enzyme by ubiquitin-chain assembly on its catalytic residue. Nat Cell Biol 2007; 9:422-427.

65 Yang G, Li QR, Ren S, et al. Proteomic, functional and motif-based analysis of C-terminal Src kinase-interacting proteins. Proteomics 2009; 9:4944-4961.

66 Rappsilber J, Ishihama Y, Mann M. Stop and go extraction tips for matrix-assisted laser desorption/ionization, nanoelectrospray, and LC/MS sample pretreatment in proteomics. Anal Chem 2003; 75:663-670.

(Supplementary information is linked to the online version of the paper on the Cell Research website.) 